Elsevier required licence: (C) 2018

This manuscript version is made available under the CC-BY-NC-ND 4.0 license http://creativecommons.org/licenses/by-nc-nd/4.0/

The definitive publisher version is available online at $\underline{\text { 10.1016/j.apenergy.2017.11.071 }}$ 


\title{
An Analysis-Forecast System for Uncertainty Modeling of Wind Speed: A Case Study of Large-scale Wind Farms
}

JianzhouWang ${ }^{a}$, Tong $\mathrm{Niu}^{a},{ }^{*}$, Haiyan $\mathrm{Lu}^{b}$, ZhenhaiGuo $^{c}$, WendongYang ${ }^{a}$, Pei Du ${ }^{a}$

${ }^{a}$ School of Statistics, Dongbei University of Finance and Economics, Dalian 116025, China.

${ }^{b}$ Department of Software Engineering, University of Technology, Sydney, Australia.

${ }^{c}$ State Key Laboratory of Numerical Modelling for Atmospheric Sciences and Geophysical Fluid Dynamics, Institute of Atmospheric Physics, Chinese Academy of Sciences, Beijing 100029, China.

* Corresponding author. Address: School of Statistics, Dongbei University of Finance and Economics, Shahekou district, Dalian 116025, Liaoning province, China. Tel.:+86 18340831005;

\begin{abstract}
The uncertainty analysis and modeling of wind speed, which has an essential influence onwind power systems, is consistently considered a challenging task. However, most investigationsthus far were focusedmainly on point forecasts, which in reality cannot facilitate quantitative characterization of the endogenous uncertainty involved.An analysis-forecast system that includes an analysis module and a forecast module and can provide appropriate scenarios for the dispatching and scheduling of a power system is devised in this study; this system superior to those presented in previous studies.In order to qualitatively and quantitatively investigate the uncertainty of wind speed, recurrence analysis techniques are effectively developedfor application in the analysis module. Furthermore, in order to quantify the uncertainty accurately, a novel architecture aimed at uncertainty mining is devised for the forecast module, where a non-parametric model optimized by animproved multi-objective water cycle algorithm is considered a predictor for producing intervals for each mode component after feature selection. The results of extensive in-depthexperiments showthat the devised systemis not only superior to the considered benchmark models, but also has good potential practical applications in wind power systems.
\end{abstract}

Key Words: Analysis-forecast system; Chaos technique; Multi-objective optimization algorithm; Feature selection; Wind speed series

\section{Introduction}

In recent years, given its advantages, such as renewability and cleanness, the comprehensive exploitation and utilization of wind energy has made it extensively socially and economically effective.More importantly, it is self-evident in a comparison of wind energy and conventional energy, which is a significant cause of global warming and atmospheric contamination, that wind power is one of the most promising energy sources available worldwide.Thus, wind energy is a greatly preferred energy resource in many parts of the world [1]. For example, wind power may become the second largest resource for generating electricityin China by 2050 [2]. However, in practice,theefficient and comprehensivedevelopment of wind power systemsis considerablyrestricted because ofthe intrinsic randomness and intermittency of wind speed, which presentsa significant challenge in terms ofelectrical network operation and management, in particularwind power integration (WPI). Accordingly, the effective analysis and accurate forecasting of wind speed not only constitutea challenging task, but arealso an emphatic concern for those who make decisions-related to wind farms. It is crucial bothto design more appropriate and efficient wind farms and to further determinethe nonlinear dynamic pattern of wind speed in order to better manage and minimize the operational risks.

The analysis and investigation of the dynamic characteristics, in particular the 
predictability, of nonlinear systems are important for forecast modeling. However, most of thestudies in the literature placed emphasis mainlyon certain basic statistics, such as the maximum, minimum, average, and standard deviation [3-4]. Further, the Lyapunov exponent, complexity, skewness, kurtosis, andemergence of wind speed were investigated in reference[5]. Effective studies on the statistical distribution of wind speed, which is usually assumed to be a Weibull distribution function, in order to further determine wind speed patterns were reported in references [6-8]. Evidently, these statistics do not suffice to reveal the profoundcharacteristics of complex nonlinear systems, in particular highly volatile wind speed series. The recurrence plot and recurrence quantification analysis, which is essentially based on chaos theory, as an effective techniqueforstudying complicated nonlinear systems, were developed in the field of wind speedforecasting.In the study reported in reference [9], wind speed series were analyzedusing recurrence plots. However, this analysis waslimited to recurrence plots, and is still not sufficient toquantitatively investigate the system behaviors of wind speedseries. In order to further remedy the defect of recurrence plots that they lackquantitative analyses, arecurrence quantification analysisof recurrence plots, which can also be used to visualize the trajectories in phase space, was effectively developedin this study in order to investigatein greater depththe dynamic characteristics and predictability of wind speed series and the corresponding mode components.

Accurate modeling of wind speed has important practical significance for wind energy development and utilization in many forms, such as wind turbines that convertwind power into kinetic energy and mean flow acoustic enginesthat convert the mean flow power into acoustic power [10-12]. However, given the complex dynamic pattern of wind speed, the design of an effective and scientific wind speed forecast model (WSFM) is consistently attractingconsiderable research attention.In general, the mainstream studies ofWSFMs can be systematically categorized into those using physics and statistical approaches [13] and artificial intelligence methods. Rich physics models involving wind speed forecasts (WSFs) were systematically introduced in references [14-18]. Technically, these models in general involve computational fluid dynamics in order to simulate the atmosphere based on different grid designs[19].In contrast to physics models, the alternative WSFMs are basedon statistical modeling and machine learning theories, which are convenient forimplementing the modeling and simulation of wind speed forecasting because of theiraccessibility and excellent local prediction ability.In earlier research on WSFMs, the traditional statistical models, which usually consist of an autoregressive model (AR) [20], autoregressive integrated moving average model (ARIMA) [21-23], fractional-ARIMA [24], or autoregressive conditional heteroskedasticity model (ARIMA-ARCH) [25], played a widespread role in the WSF field. In recent years, forecast models based on machine learning theories, in particular artificial neural networks (ANNs), have becomepopularin the WSF field. In general,they are trained using the historical information of wind speedin order to establish nonlinear mapping between theinput set and target set. Theoretically, the self-learning and self-organizing capabilities of ANNs are excellent and therefore, considerable effort has been invested by many researchers in ANNs for use in WSF [26-28]. However, the effectiveness and efficiency of hybrid models in generalmakes them superior tosingle neural network modelsin terms ofachieving accurateWSFs. As a consequence, many studies onhybrid forecast models have been reportedevery year. Most of thesemodels usually focused attention ondata preprocessing [29-32] and model parameter optimization using heuristic algorithms, such as theparticle swarm optimization (PSO) [33-34], 
andgenetic algorithms (GAs) [33, 35-37].

In addition to wind speed forecasting, reliable wind power forecasting plays an important role in the scheduling and operation of wind farm power systems. Many scholars have invested effort in the study of accurate wind power forecasting using models-based machine learning theory. In [38], an adaptive network-based fuzzy inference system, which incorporated a wavelet and a PSO algorithm, was developed to achieve short-term wind power forecasting. A hybrid forecasting model, combining a support vector machine (SVM) and a Markov model, was proposed in [39] to achieve wind power forecasting. In [40], a random forests model was proposed, aimed at performing one hour ahead wind power forecasting. ANNs with self-learning and generalization capabilities have been widely applied in the field of wind power forecasting. In [41], a bidirectional mechanism using an extreme learning machine (ELM), a well-known ANN model, was established for wind power forecasting. In order to achieve accurate wind power forecasting, an effective forecasting framework, including a local linear fuzzy neural network (LLFNN) optimized by a seeker optimization algorithm, discrete wavelet transform, and singular spectrum analysis, was proposed in [42]. In [43], a forecasting model based on chaotic time series was presented for wind power forecasting, where phase space reconstruction and a Bernstein neural network were combined. Additionally, effort was invested in wind power forecasting using a radial basis function neural network (RBFNN) [44] and wavelet neural network (WNN) [45] with the aim of achieving accurate wind power forecasting results.

Most of the aforementioned studies were focused mainly on point forecasts, which cannot easily quantify uncertain information in the process of wind speed forecasting. However, the study of the interval prediction of wind speed or wind power has not received sufficient attention, despite its significance to the risk management, power dispatching and WPI of wind farms. In practical power grid management, uncertainty analysis and mining is beneficial for ameliorating the adverse effects ofthe stochastic volatility of wind speed and for effectively providingmore comprehensive reference information to operational risk decision makers.Forthis reason, uncertainty modelingis becoming a prevailing research direction of many scholars in this field of the study.However, the study of uncertainty modeling is still in its infancy.Currently, there are only a few studies on uncertainty quantification, and the mainstream research direction relies largely on statistic methods, including quantile regression [46-48], bootstrap methods[49], and kernel density estimation [50]. Additionally, an interval prediction method using nonparametric theory, lower upper bound estimation (LUBE), based on ANNswas proposed to construct prediction intervals [51].

A comprehensive evaluation of the forecasting models for wind speed and wind power mentioned above was conducted in this study; the results are summarized in Table 1. In point forecasting models, the application of physics models is significantly restricted because of the complex meteorological conditions, model initialization, and heavy computation cost, despite their excellent long-term forecasting capabilities. The computation efficiency of conventional statistical models, including AR, ARIMA, and so forth, is high. However, their linear form restricts their ability to model accurately nonlinear time series, such as wind speed and wind energy time series. A key problem related to ANNs is that they are easily trapped in local optimization, although they have excellent capabilities for modeling nonlinear time series. Furthermore, in the field of interval prediction, research is focused on quantile regression because of its particular advantages, shown in Table 1. However, the main drawback of quantile 
regression methods is that it is necessary to acquire a particular training dataset to 2 establish a forecast model when using the method to develop prediction intervals. 3 Additionally, in quantile regression each quantile needs to be modeled, which 4 increases not only the computational burden but also the probability of results being 5 discarded in the resampling process [52].The bootstrap method is a statistical method 6 that uses data resampling with replacementto estimate the robust properties of 7 almostany statistics, such as standard errors, some parameters of confidence intervals, 8 and the coefficients of correlation and regression [53]. Bootstrap methods can avoid 9 the possible drawbacks of the quantile regressionmethod. However, they are only 10 veryeffective when addressing small sample sizes and thustheirapplication isrestricted 11 when addressing a large-scale sample set. Kernel density estimation, which can 12 construct prediction intervals rapidly, is based on point forecast results alongwith an assumed statistical, usually Gaussian, distribution of historical errors.However, the presumed error distribution does not matchthe actual error distribution. Accordingly, merely using Gaussian distribution to configure the error distribution is far fromsufficient. Considering the aforementioned analysis, the hypothetical error distribution using Gaussian distribution may unavoidably produce the biasand risks when developing prediction intervals.As compared withtraditional interval prediction models based on parameter statistics, theLUBE method avoids the restrictive distribution assumption and heavy computation burden when constructing prediction intervals. However, the objective function construction of the LUBE method is complex and cannot be optimized by using traditional mathematical methods. In this study, an improved multi-objective optimization algorithm was employed to optimize the key parameters of the LUBE method, which is an additional contribution of this study.Existing progress inWSFM using LUBE was achieved mainly with the aid of ANNs. However, ANNs are sensitive to complex training parameters and likely to become trapped inlocal optima. Accordingly, a robust multi-input multi-output least squares SVM (MIMOLSSVM) based on machine learning theory, which requires fewer parameters that need to be tuned than NNs, was developed in this study.

Table 1. Evaluation of forecasting models including point and interval prediction.

\begin{tabular}{|c|c|c|c|}
\hline Category & Model & Merit & Demerit \\
\hline \multirow{3}{*}{ Point prediction } & Physics models & $\begin{array}{l}\text { Good space-time continuum; high } \\
\text { temporal and spatial resolution; } \\
\text { clear physics process; long-term } \\
\text { forecasting. }\end{array}$ & $\begin{array}{l}\text { Complex modeling process; heavy } \\
\text { computational burden; poor local } \\
\text { predictability; large forecasting } \\
\text { error resulting from complex } \\
\text { meteorological conditions and } \\
\text { model initialization. }\end{array}$ \\
\hline & $\begin{array}{l}\text { Statistical models (AR, } \\
\text { ARIMA, ARIMA-GRCH, } \\
\text { fractional-ARIMA.) }\end{array}$ & $\begin{array}{l}\text { High computation efficiency; less } \\
\text { model parameters to be tuned; good } \\
\text { predictive performance for linear } \\
\text { data. }\end{array}$ & $\begin{array}{l}\text { Poor prediction accuracy for } \\
\text { nonlinear data; applicable only to } \\
\text { stable data; assume that the } \\
\text { interference sequence is white } \\
\text { noise. }\end{array}$ \\
\hline & $\begin{array}{l}\text { Artificial neural network } \\
\text { (such as WNN, RBFNN, } \\
\text { ELM, and so forth.) }\end{array}$ & $\begin{array}{l}\text { Able to approximate any nonlinear } \\
\text { relationship theoretically; good } \\
\text { generalization capability; excellent } \\
\text { self-learning capability. }\end{array}$ & $\begin{array}{l}\text { Complex computational process; } \\
\text { sensitive to the size of training } \\
\text { samples; easily fall into the local } \\
\text { optimum. }\end{array}$ \\
\hline \multirow{3}{*}{ Interval prediction } & Quantileregression & $\begin{array}{l}\text { Able to handle heterogeneity } \\
\text { problem; not sensitive to outliers; } \\
\text { considers the entire distribution; } \\
\text { able to capture the tail } \\
\text { characteristics of the distribution. }\end{array}$ & $\begin{array}{l}\text { Requires a specific set of training } \\
\text { samples; heavy computational } \\
\text { burden; the probability of results } \\
\text { being discarded in the process of } \\
\text { repetitive computing. }\end{array}$ \\
\hline & Bootstrap methods & $\begin{array}{l}\text { Avoid possible discards in quantile } \\
\text { regression; very effective when } \\
\text { dealing with small samples. }\end{array}$ & $\begin{array}{l}\text { Poor performance when handling } \\
\text { large samples; heavy computational } \\
\text { burden. }\end{array}$ \\
\hline & Kernel density estimation & $\begin{array}{lll}\begin{array}{l}\text { Easily } \\
\text { interval. }\end{array} & \text { constructs } & \text { prediction } \\
\end{array}$ & Strict assumptions on distribution. \\
\hline
\end{tabular}


Avoids the assumptions about Complex objective function; the distribution of studied data; high objective function cannot be computational efficiency; easily optimized by the traditional adjustable model coefficients.

mathematical method.

Most of the literature concerning wind speed forecasting underlines mainly data preprocessing and model optimization. The investigation of feature selection as applied in wind speed forecasting has received little attention. Feature selection, which can remove certain irrelevant features and enhance the capability of the forecasting model to learn the nonlinear relationship in time series, is an effective technique for selecting appropriate model input when performing forecasts. In previous studies in the literature, the input forms of the model usually depended on subjective experience and repeated experiments, which reduces to a certain degree the efficiency of constructing prediction intervals. The development of a feature selection technique for interval prediction models of wind speed is an important contribution of this study. Furthermore, more effort should be invested in developing feature selection for wind speed and power forecasting to improve its accuracy and efficiency further.

In consideration ofthe significance of nonlinear analysis and forecast modeling, a novel analysis-forecast system, combiningananalysis module and aforecast module, is proposed in thispaper. For theanalysis module, recurrence analysis techniques based on chaos theory, including recurrence plot and recurrence quantification analysis, were effectively developed to study the dynamic behaviors and predictability of the nonlinear system based on wind speed series. For the forecast module, a novel framework of uncertainty mining was devised, which systematically combines LUBE theory, MIMOLSSVM, complete ensemble empirical mode decomposition with adaptive noise (CEEMDAN) based on mode decomposition theory, afeature selection technique using phase space reconstruction, and an improved multi-objective water cycle algorithm (IMOWCA). However, MIMOLSSVM is also sensitive to the inherent parameters, namely regularization parameter and squared kernel bandwidth parameter.IMOWCA, aimed to optimize the key parameters of the forecast module in order to strengthen the effectiveness and robustness of MIMOLSSVM, is presented for the first time in this paper. In fact, feature selection can enhance the operational efficiency by reducing the training time and improve the model generalization by avoiding over-fitting. However, in previous studiesof interval prediction, the feature selection technique usually was not taken into account in the development of uncertainty modeling. In this study, a classical and effective feature selection technique based on chaos theory, the C-Cmethod,was developed for implementing feature selection and thus obtaining the optimal input forms forMIMOLSSVM. More importantly, in order to effectively model the nonlinear system based on wind speed series, the raw wind speed series is decomposed intointrinsic mode functions (IMFs) by using theCEEMDAN method. Furthermore, in order to reduce the computation complexity, the generated IMFs are mergedin accordance with the correspondingcomplexity degree, and then, the proposed model implements uncertainty modeling for each reconstituted IMF. Finally, the prediction intervals generated by each IMF are mergedto obtain the final interval prediction results. The devised analysis-forecast system is calledModes-IMOWCA-CC-MIMOLSSVM, accordingly.

In contrast to aparametric model, the devised forecast module based on LUBE makes no assumption concerning distribution shape, and thus, uncertainty modelingismore convenient and effective. As compared to NNs, the forecast module based on MIMOLSSVM needs fewermodel parameters and avoids the over-fitting problem, usually obtaining satisfactory forecast results. Phase space reconstruction 
based on chaos theory, which is superior to the previous feature selection methods, was developed in this studyto adaptively determine the optimal inputfeature.

The main contributions of the devised analysis-forecast system can be summarized as follows.

(1) A novel analysis-forecast system of wind speed is proposed in this paper,aimed at improving the effectiveness of constructing effective prediction intervals to improve the management and scheduling of wind power systems.

(2) The notion of mode components wasoriginally developed in this study with the aim of effectively performing uncertainty analysis and mining for the nonlinear system based on wind speed series, which is proved to be an effective and robust method.

(3)Importantly, the particularadvantage of the devised forecast module is its simplicity, since it avoids the assumption on distribution shape, as compared to conventional parametric statistical models. Thissignificantly reduces the complexity of uncertainty modeling and strengthens the robustness and efficiency of the system.

(4)The feature selection technique based on delayed embedding theorywasdeveloped in this study to determine the optimal input features when developing the prediction intervals, which is an important contribution of this study.

(5)Together with phase space reconstruction, the inherent trajectories of anonlinear system based on wind speed series aredeterminedusing recurrence plots and recurrence quantification analysis, which can effectively reveal the predictability of wind speed series.

(6)IMOWCA is proposed in this paper to optimize the key parameters of the forecast module in this system. The experimental results manifest that IMOWCA outperforms its primitive in the process of constructing prediction intervals.

(7)Effective sensitivity testing, which further elucidates the robustness, effectiveness, and efficiency of the devised analysis-forecast system, is described in this paper, and extensive discussions are presented.

The remainder of this paper is organized as follows. Section 2 introduces the preliminaries of the proposed analysis-forecast system. In Section 3, the overall framework of the system is introduced. Implementations of the analysis module and forecast module to verify the effectiveness of the proposed system are described in Section 4. Further discussions about the system are presented in Section 5. Finally, the conclusion of this paper is put forth in Section 6.

\section{Methodology}

In this section, a modes decomposition method and recurrence analysis techniques are introduced. Furthermore, the detailed theory of feature selection using the C-C method is described. Finally, MIMOLSSVM and its optimization using IMOWCA are introduced.

\subsection{Modes Decomposition andRecurrence Analysis}

Nonlinear systems, in particular wind speed series, have complex system characteristics, such as high volatility, randomness and intermittency, and thus, it is difficult to accurately model the uncertaintyof wind speed series. Therefore, mode (or frequency domain) decomposition for wind speed series, which can largely reduce their complexity, must be implemented. In this section, a novelmode decomposition method, namely CEEMDAN, isbriefly introduced. Additionally, considering the complexity of wind speed series, an effective frequency-time analysis modulebased on recurrence plots and recurrence quantification analysisis used to analyze and study the dynamic characteristics of wind speed series.

In order to effectively investigate and model the frequency components of wind 
speed, CEEMDAN, a powerful mode decomposition method, isapplied in the devised forecast module. CEEMDAN, an advanced extension of the complementary ensemble empirical mode decomposition (CEEMD) method proposed by Jia-rongYeh et al. in [54], was first presented in [55]. As compared to the previously proposed empirical mode decomposition (EMD) [56], ensemble empirical mode decomposition (EEMD) [57], and complementary ensemble empirical mode decomposition (CEEMD) methods, the distinct merits of CEEMDAN are as follows. (1) The noise coefficient vector is extended to adjust the added noise level in the process of decomposition. (2) The generated IMFs are completely reconstructed without a noise component. (3) The method is more efficientthan EEMD and CEEMD. Further details concerning CEEMDAN can be found in reference [55].

The nonlinear systems, in particularwind speed series,show significant uncertainties, unexpected randomness, and complicated nonlinear specialties, and thus, uncertainty modeling is a challenging task. Therefore, explorations of the systematic features of nonlinear systems are always constantly in progress worldwide. Recurrence is a fundamental property of adynamical system, which can be exploited to characterize the system's behavior in phase space [58]. In general, therecurrence phenomenon occursin nonlinear systems, especially chaos systems, which provides an effective path for investigating the dynamic properties based on phase space constructedby the C-Cmethod.Thus, therecurrence plot was first proposed by Eckman et al. [59] in order to effectively address the problem. The recurrence plot can be implemented via the following matrix $\mathbf{R}_{i, j}$, which can betranslated into arecurrence plot.

$$
\mathbf{R}_{i, j}=\Theta\left(\varsigma-\left\|\stackrel{\mathrm{r}}{x_{i}}-\stackrel{\mathrm{r}}{x_{j}}\right\|\right), i, j=1,2, \mathrm{~L}, N(1)
$$

where $\vec{x}_{i}$ denotes a point in phase space, and $\varsigma$ and $\Theta$ represent the threshold and Heaviside function, respectively. It is worth mentioning that the threshold is in generaldetermined as 0.4-0.5 times the standard deviation of the studied wind speed data.

Table 2 [58] showsidentification methods of recurrence plotsand their different interpretations.In Fig. 1, an illustrative examplebased on theLorentz system in order not only to visualize the recurrence plot, but also toanalyze its characteristicsis shown. Inthis example, the embedding dimension and delay time of Lorentz series $\left(X_{i}, i=1,2, \mathrm{~L}, 3000\right)$ can be obtained by the C-Cmethodmentioned below. According to the obtained embedding dimension and delay time, the Lorentz attractor can be retrieved, as shown in Fig. 1.In this figure, the recurrence plot is clearly displayed with athreshold value 0.3 for the recurrence matrix, from which the conclusion can be drawnthat the analyzed system is chaotic according to the sixth identification method in Table 2.

Table 2. Identification approaches forrecurrence plots.

\begin{tabular}{ll}
\hline Observation & Interpretation \\
\hline Homogeneity & $\begin{array}{l}\text { Stationary process. } \\
\text { Fading to the upper left and lower right corners }\end{array}$ \\
& $\begin{array}{l}\text { Nonstationarity; some states are rare or escape } \\
\text { the normal; transitions may have occurred. }\end{array}$ \\
Tapebreak occurs & $\begin{array}{l}\text { Nonstationarity; states are rare or far from the } \\
\text { normal. }\end{array}$ \\
Periodic or quasi-periodic patterns & $\begin{array}{l}\text { Periodical process; long diagonal lines with } \\
\text { different distances between each other reveal a } \\
\text { quasi-periodic process. }\end{array}$ \\
\hline
\end{tabular}


Single isolated points

Heavy fluctuation in the process; the appearance of single isolated points impliesrandomness in the process.

Diagonal lines

Deterministic process; the evaluation of states is analogical at differenttimes; the process is chaotic if diagonal lines occur alongside single isolated points.

Vertical and horizontal lines/clusters $\quad$ Weak volatility in the process; laminar states have occurred.

\section{have occurted.}

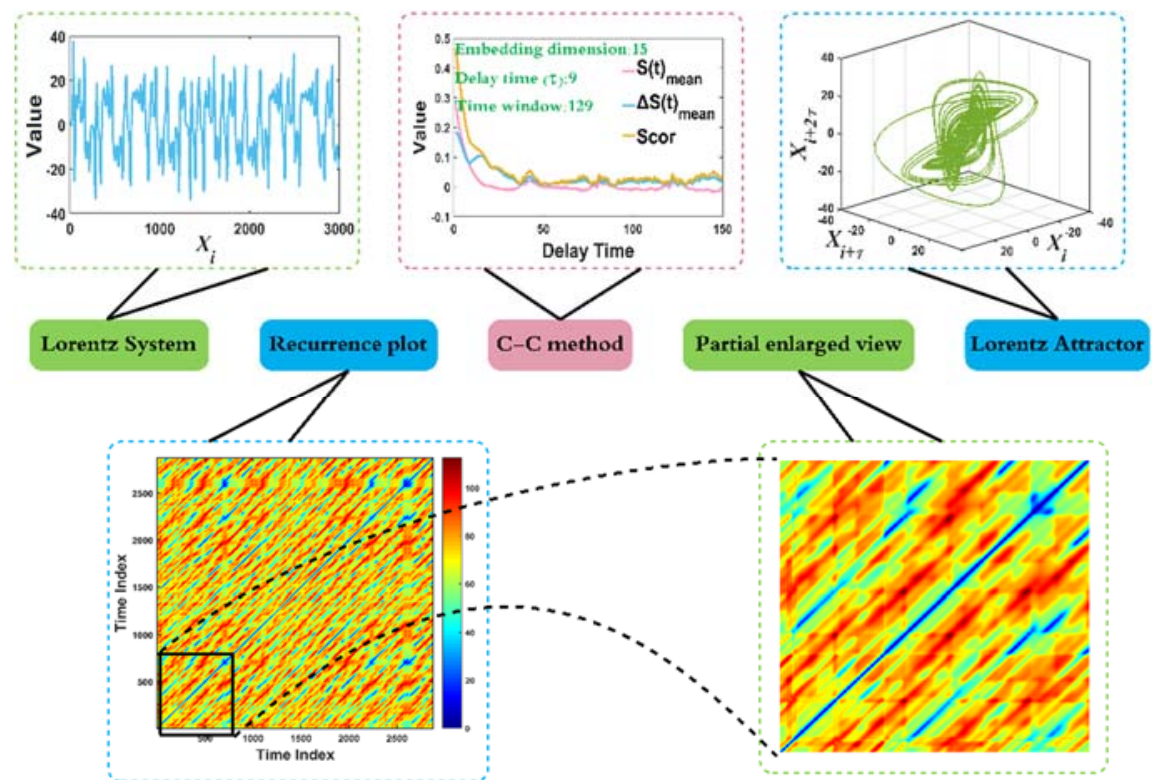

Fig. 1. Illustrative example of recurrence plot.

In this study, recurrence plots,togetherwith CEEMDAN,were used to unveil the intricate dynamic traits of anonlinear system and visualize the trajectories existing in actual phase space. Theoretically and technically, the times when the trajectory encounters approximately the same region in phase space can be effectively identified by the recurrence plot.

Nevertheless, merely using the recurrence plot to identify the dynamic pattern of anonlinear system is notsufficientbecause ofthe absence of aqualitative analysis.Forthis reason, arecurrence quantification analysis including metrics was proposed in [60-62] in order to analyze a nonlinear system from the qualitative perspective, which usually involves certainstatistical standards, including recurrence rate $(R R)$, determinism $(D E T)$, entropy $(E N T R)$, andaverage diagonal line length $(L)$.The definitions of these four metrics are described as follows.

(1)Recurrence rate $(R R): R R$ is a metric that calculates the proportion of recurrence points corresponding to the recurrence plot, which can be utilized to uncover the system dynamics in phase space.

$$
R R(\varsigma)=\frac{1}{N^{2}} \sum_{i, j=1}^{N} \Theta\left(\varsigma-\left\|\stackrel{\mathrm{r}}{x_{i}}-\stackrel{\mathrm{r}}{x_{j}}\right\|\right), i \neq j(2)
$$

At least 5 meters in length

(2)Determinism (DET): DET is the ratio of recurrence points that form diagonal structures of length at least $l_{\min }$ toall recurrence points. Theoretically, the phenomenon ofno or very short diagonals occurs if the processesareuncorrelated or weaklycorrelated and the behavior is stochastic or chaotic, whereas adeterministic process produces longerdiagonals with fewersingleisolated recurrence points. 
Accordingly, DET provides an insight for investigating the determinism andthe predictingcapabilityof a system.

$$
D E T=\frac{\sum_{l=l_{\min }}^{N} l P(l)}{\sum_{l=1}^{N} l P(l)}(3)
$$

where $P(l)$ is the histogram of diagonal lines of length $l$ with the threshold $\varsigma$.

(3)Average diagonal line length $(L): L$ is the average distance betweentwo segments of the trajectory, which can be interpreted as the mean prediction time.

$$
L=\frac{\sum_{l=l_{\min }}^{N} l P(l)}{\sum_{l=l_{\min }}^{N} P(l)}(4)
$$

(4)Entropy $(E N T R)$ : ENTR refers to the Shannon entropy of the probability $\mathrm{p}(l)=$ $P(l) / N_{l}$ to find a diagonal line of exactly length $l$ inthe recurrence plot, which reflects the complexity of the recurrence plot with respect to the diagonal lines.

$$
E N T R=-\sum_{l=l_{\min }}^{N} p(l) \mathbf{I n} p(l)(5)
$$

\subsection{Feature Selection}

The effective modeling of complex nonlinear systemshas always been a hot topic in the academic community of nonlinear or complex systems. Detection of the immanent mechanism and dynamics is of great significance for modelinganonlinear system, and this led to the birth of phase space reconstruction. Feature selection through a classical phase space reconstruction technique, namely, theC-C method based on chaos theory, which was proposed by Kim H S[63], was developed in this study. In the process of phase space reconstruction, accuratedetermination of the optimal delay time $(\tau)$ and embedding dimension $(m)$ is of crucial significancefor retrieving the attractor in high-dimensional phase space. Further details ofphase space reconstruction based on the $\mathrm{C}-\mathrm{C}$ method are providedin the following.

Consider a time series $\left\{x_{j} \mid j=1,2, \ldots, j\right\}$. The phase space can be accurately reconstructed in accordance with the aforementioned parameters $\tau$ and $m$, respectively:

$$
X_{i}=[x(i), x(i+\tau), \cdots, x(i+(m-1) \tau)](6)
$$

It is noteworthythat thephase space reconstruction technique provides a new perspective for analyzing the nonlinear system. However, improper, or even inaccurate, determinations of $\tau$ and $m$ will lead to significantly negative influenceson the effectiveness of theforecast model, such as anunsatisfactory forecast accuracy and potential management risk. The process of phase space reconstruction based on the $\mathrm{C}-\mathrm{C}$ method comprises the following five steps.More information ofthe $\mathrm{C}$-Cmethod can be found in reference [63].

(1)Determine the suitable length of the time series and then calculate its standard deviation;

(2)Calculate the metrics $S(t)_{\text {mean }}$ and $\Delta S(t)_{\text {mean }}$.

$$
\begin{gathered}
S(t)_{\text {mean }}=\frac{1}{16} \sum_{m=2}^{5} \sum_{j=1}^{4} S\left(m, r_{j}, \tau\right)(7) \\
\Delta S(t)_{\text {mean }}=\frac{1}{4} \sum_{m=2}^{5} S(m, t)(8)
\end{gathered}
$$

where $r_{j}=j \delta / 2, j=1,2, \ldots, 4$.

(3)Determine the optimal delay time when $S(t)_{\text {mean }}$ first reaches zero or first 
reaches the minimum value.

(4)Coupling the metrics $S(t)_{\text {mean }}$ and $\Delta S(t)_{\text {mean }}$, the statistic $S c o r(t)$ can be obtained when $\operatorname{Scor}(t)$ reaches the global minimum, which can be calculated according to:

$$
\operatorname{Scor}(t)=\Delta S(t)_{\text {mean }}+\left|S(t)_{\text {mean }}\right|(9)
$$

(5) The optimal time window $\varpi$ can be determined when $\operatorname{Scor}(t)$ reaches the global minimum value. Furthermore, the $m$ can be obtained via the Eq. (10).

$$
\varpi=(m-1) \tau(10)
$$

\subsection{Multi-input Multi-output Least Squares Support Vector Machine}

In this section, a classical machine learning model MIMOLSSVM, which is applied to perform the interval prediction, is introduced.In addition, the proposedIMOWCA to further optimize the performance of MIMOLSSVMis described.

The MIMOLSSVM model, based on the principle of structural risk minimization [64-66], is a powerful tool for implementing interval prediction via the multi-output pattern. However, the application of MIMOLSSVM to uncertainty modeling is rarely implemented, despite the fact thatit has excellent nonlinear system modeling capabilities, in particular for uncertainty mining.

Consider the training dataset as $\boldsymbol{T}=x_{i}, y_{i}{ }^{n}$, where $x_{i}$ belongs to $\square^{p}, y_{i}$ belongs to $\square^{d}$, and $x_{i}$ and $y_{i}$ represent the input and output dataset of the training set, respectively. Additionally, $\square^{p}$ denotes the input space with the dimension of $p$; the dimension $p$ is optimally and dynamically determined according to the obtained embedding dimension via theaforementioned $\mathrm{C}-\mathrm{C}$ method, and $\square^{d}$ is selected as the value of 2 considering the prediction interval with the upper and lower bound in this study. Technically, the classical LSSVM can be formulated as:

$$
y=w^{T} \phi(x)+b(11)
$$

wherew and $b$ denote the weights and the bias, respectively, and $\phi$ signifies the function mapping stemming from the nonlinear relationship between input and output sets.More details of LSSVM can be found in reference [67].

The classical LSSVM model has anexcellentability to model the nonlinear series with the pattern of single-output. However, the LSSVM model with single output does not evidently meet the requirement that interval prediction be implemented, leading to inferior forecast results, even if two or more single LSSVM models are combinedinto a multi-output LSSVM, because this overlooksthecombined fitting bias generated by multiple LSSVM models. Accordingly, MIMOLSSVM wasdevelopedin this studyto perform the interval prediction. The detailed theory of MIMOLSSVM can be found in reference [68].

\subsection{Introduction to the Improved Multi-objective Water Cycle Algorithm}

In this section, the flow of the original water cycle algorithm (WCA) is introduced. Furthermore, the improved multi-objective watercycle algorithm (IMOWCA), aimed at optimizing the devised forecastmodule, is proposed. It is described as follows.

\subsubsection{Water Cycle Algorithm}

Inspired by the actual water cycle process, the single-objective WCA, which is extensively applied in many fields, such as electrical power system [69-70] and traffic light scheduling [71], was proposed by Eskandaret al. [72].The calculation process ofthe WCAisas follows. The initial population of size $N_{p o p}$ can be obtainedrandomly.It isdivided into two sections in accordance with the fitness values. The first section consists of $N_{s r}$ raindrops, which have a better fitness thanthe second section. The best 
raindrop and some rivers are grouped in the first section. The second section is composed ofmany raindrops, which are calledthe streams in this algorithm. The size of thestreams, which are allocatedto theaforementioned first section, can be calculated according to Eq. (12), where $\boldsymbol{C o s t}_{n}$ represents the fitness value of the $n$-th raindrop.

$$
N S_{n}=\operatorname{round}\left\{\left|\frac{\text { Cost }_{n}}{\sum_{k=1}^{N_{s r}} \boldsymbol{C o s t}_{k}}\right| \times\left(N_{p o p}-N_{s r}\right)\right\}, n=1,2, \cdots, N_{s r}
$$

Further, the algorithm consists of the following steps.

Step 1:The iterative process of the new positions of streams and rivers $\left(\overrightarrow{\mathbf{X}}_{\text {Stream }}^{i+1}\right.$, $\overrightarrow{\mathbf{X}}_{\text {River }}^{i+1}$ ) can be expressed as Eqs. (13)-(15), which describe how the streams and rivers move toward sea while updating their positions. It isnoteworthy that the optimum determination of $C$ is 2, which was proposed in [69]; rand is a value that obeys the uniform random distribution in the range $[0,1]$.

$$
\begin{aligned}
\dot{X}_{\text {Stream }}^{i+1} & =\grave{X}_{\text {Stream }}^{i}+\operatorname{rand} \times C \times\left(\mathbf{X}_{\text {River }}^{i}-\grave{X}_{\text {Stream }}^{i}\right)(13) \\
\mathbf{X}_{\text {Stream }}^{i+1} & =\mathbf{X}_{\text {Stream }}^{i}+\operatorname{rand} \times C \times\left(\mathbf{X}_{\text {Sea }}^{i}-\mathbf{X}_{\text {Stream }}^{i}\right)(14) \\
\mathbf{X}_{\text {River }}^{i+1} & =\mathbf{X}_{\text {River }}^{i}+\operatorname{rand} \times C \times\left(\mathbf{X}_{\text {Sea }}^{i}-\mathbf{X}_{\text {River }}^{i}\right)(15)
\end{aligned}
$$

Step 2:The positions of each river and stream are automatically exchangedwhen the fitness of thestream is better than that ofthe rivers. Similarly, the position of thesea is replaced with its assigned stream or river when their fitness value is greater than that of the sea.

Step 3:The behavior of evaporation and precipitation istriggered on condition that the evaporation condition, as shown in Eq. (16), is satisfied. Consequently, the position of streams is initialized, leading to the new positionsof streamsaccording to Eq. (17). Furthermore, the optimal position of astream is considered to be the river that flows toward the sea. Analogically, the new position of the stream can be calculated according to the stream that flows to the seaif the evaporation condition is satisfied, shown in Eq. (18). The noteworthy point is that the operation of evaporation can reduce the probability that the algorithm falls prematurely into local optima.

$$
\begin{aligned}
& \left|\mathbf{X}_{\text {Sea }}^{i}-\mathbf{X}_{\kappa}^{i}\right|<d^{i}{ }_{\text {max }}, \kappa \in\{\text { Stream, River }\} \text { (16) } \\
& \dot{\mathbf{X}}_{\text {New Stream }}^{i}=\mathbf{L B}+\operatorname{rand} \times(\mathbf{U B}-\mathbf{L B})(17)
\end{aligned}
$$

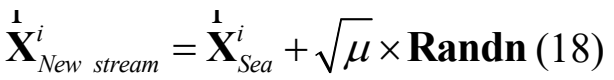

where $d_{\max }$ is set as $10^{-6}$, andRandn is a random vector that obeys uniform distribution with the range $[-1,1]$. Additionally, LB and $\mathbf{U B}$ represent the lower and upper bounds of variables. Finally, $\mu$ was set as 0.1 inthe study[69].

Step 4:The tolerance in the evaporation condition, namely $d_{\max }$, adaptively decreases in the process of iteration, which isshown in

$$
d_{\max }^{i+1}=d_{\max }^{i}-\frac{d_{\max }^{i}}{\text { max_iteration }}
$$

Step 5:The algorithm isfinalizedif the end condition (such as maximum iteration numbers) is satisfied;Otherwise, it returns toStep 1.

\subsubsection{Improved Multi-objective Water Cycle Algorithm}

The innovation of the IMOWCA proposed in this paper is that the adaptive and 
nonlinear inertia weight $(\omega)$, which has an excellent capability to balance the global and local search capability in the process of algorithm iterations, as shown in Eq. (20), is introduced into the original MOWCAfor the first time. Technically, when $\omega$ is large, the IMOWCA has an excellent global exploration capability, while its local exploitation ability is poor; conversely, the IMOWCA has noteworthysuperiority in terms of local exploitation, while its global exploration ability is poor. Accordingly, determining the appropriate $\omega$ can balance the capability of global exploration and local exploitation in IMOWCA, which can significantly promote the convergence rate and effectiveness of MOWCA. The improved iteration formulations on the new position of streams and rivers in IMOWCA can be formulated as inEq. (21). Additionally, as well as the detailed calculation process of the MOWCA, the reports in [69, 73-74]provide largeamounts of information about this algorithm.

$$
\begin{aligned}
& \left\{\begin{array}{l}
\omega_{j}=\omega_{\text {end }}+\left(\omega_{\text {start }}-\omega_{\text {end }}\right) \times \exp (-3.5 \times(j / \text { max_iteration }))^{3} \\
j=1,2, \cdots, \text { max_iteration }
\end{array}\right.
\end{aligned}
$$

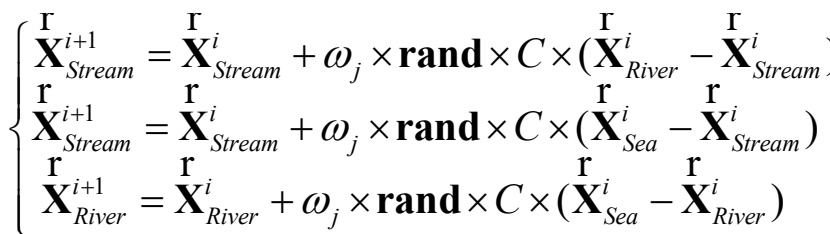

The complexity of IMOWCA isas follows. The complexity of IMOWCA is $O\left(N_{p o p}^{2}\right)$ in the worst scenario. The detailed computation processes are as shown in

Table 3, where $M$ denotes the number of objective functions and $N_{p o p}$ represents the population size in IMOWCA.

Table 3. Complexity analysis of improved multi-objective water cycle algorithm.

\begin{tabular}{ll}
\hline Algorithm Procedures & Complexity \\
\hline Determination of the sea & $O\left(N_{p o p}^{2}\right)[75]$ \\
Move streams and rivers & $O\left(N_{p o p}\right)[75]$ \\
Replace rivers and sea by better streams and & $O\left(N_{p o p}\right)[75]$ \\
rivers, respectively & $O\left(N_{p o p}\right)[75]$ \\
Check the evaporation condition & $O\left(M\left(3 N_{p o p}\right)^{2}\right)[76]$ \\
Non-dominated sorting & $O\left(M\left(3 N_{p o p}\right) \log \left(3 N_{p o p}\right)\right)[76]$ \\
Crowding distance assignment & $O\left(M\left(3 N_{p o p}\right) \log \left(3 N_{p o p}\right)\right)[76]$ \\
Rank-crowd sorting procedure &
\end{tabular}

In order to compare the proposed IMOWCA with other multiobjective optimization algorithms, a literature reviewon the subject of the complexity measurement of multi-objective optimization algorithms was conducted. The complexity of the considered multi-objective optimization algorithms is as follows. The complexity of NSGA-II [76], SPEA2 [77] and PAES [78] is $O\left(M N_{p o p}^{2}\right)$ and the complexity of NSGA [79] and SPEA [80] is $O\left(M N_{p o p}^{3}\right)$. Obviously, the IMOWCA has a lower complexity than these algorithms, which indicates that its computational efficiency is high as compared to that of these benchmark algorithms.

Importantly, the IMOWCA was developed in this study to dynamically optimize the parameter configuration in the forecast module, with the aim ofimproving the efficiency and effectiveness of the devised forecast module.

2.4.3Testing of Improved Multi-objective Water Cycle Algorithm 
In order to validate the effectiveness and efficiency of IMOWCA ascompared to MOWCA, the four testing problemsdescribedin Appendixwere performed on the platform of MATLAB R2015b on MicrosoftWindows 7 with $3.30 \mathrm{GHz}$ Intel Core i5-4590 HQ 64-bit and 8 GB of RAM. The algorithm parameters of IMOWCA and MOWCA are displayed in Table 4. Additionally, in order to obtain robust and effective simulation results, each algorithm was repeatedly simulated 20 times, and then, the final resultswere obtained by averagingthe obtained results.Generational distance (GD) [81-82] and spacing (SP) [83-84] were applied to quantitatively evaluate the performance of the two algorithms. The GD, proposed in [81], is used to measure the distance between thetrue Pareto front and obtained Pareto front. Accordingly, the smaller the value of GD, the better the performance of themulti-objective algorithm. The SP is usually applied to evaluate the distributivity of solutions in aPareto set.All non-dominant solutions are equidistant (or even) ifthe SP is equal to 0. In Table 5, the final simulation results are displayed, from which it can be concluded that IMOWCA is significantly superior to the original MOWCA on balance. The efficiency of IMOWCA is slightly superior to that of original MOWCA in the problems of ZDT1, ZDT3, andKursawe,according to the computation timesshownin Table 5. In order to further illustrate the comparativeperformance ofIMOWCA and MOWCA, the corresponding Pareto fronts obtained by IMOWCA and MOWCA arevisualized in Fig. 2, in which it can be observed that the Pareto front obtained by IMOWCA is closer to the true Pareto front than that obtained byMOWCA.

Table 4.Parameter settings of the improved multi-objective water cycle algorithm andthe multi-objective water cycle algorithm.

\begin{tabular}{ccc}
\hline Parameter Configuration & IMOWCA & MOWCA \\
\hline Population size & 200 & 200 \\
Size of archive & 100 & 100 \\
Maximum iteration & 200 & 200 \\
Number of streams & 196 & 196 \\
Number of rivers and seas & 4 & 4 \\
Evaporation condition constant & $1 \times 10^{-2}$ & $1 \times 10^{-6}$ \\
Initial value of inertia weight $\omega_{\text {start }}$ & 0.9 & - \\
Terminal value of inertia weight $\omega_{\text {end }}$ & 0.4 & - \\
\hline
\end{tabular}

25

26 27

Table 5. Assessment results of improved multi-objective water cycle algorithm and the multi-objective water cycle algorithm.

\begin{tabular}{|c|c|c|c|c|c|c|c|c|c|c|c|c|}
\hline \multirow[t]{2}{*}{ Problem } & \multirow[t]{2}{*}{ Algorithm } & \multirow{2}{*}{$\begin{array}{c}\text { CPU time } \\
\text { (s) }\end{array}$} & \multicolumn{5}{|c|}{ GD } & \multicolumn{5}{|c|}{ SP } \\
\hline & & & Best & Average & Median & Worst & Std. & Best & Average & Median & Worst & Std. \\
\hline \multirow{2}{*}{ ZDT1 } & IMOWCA & 29.7280 & 0.0015 & 0.0039 & 0.0030 & 0.0124 & 0.0026 & 0.0619 & 0.0796 & 0.0801 & 0.0933 & 0.0081 \\
\hline & MOWCA & 29.8663 & 0.0013 & 0.0094 & 0.0030 & 0.0896 & 0.0189 & 0.0151 & 0.0740 & 0.0769 & 0.0979 & 0.0169 \\
\hline \multirow{2}{*}{ ZDT3 } & IMOWCA & 29.4013 & 0.0049 & 0.0064 & 0.0062 & 0.0085 & 0.0014 & 0.1705 & 0.2066 & 0.2034 & 0.2808 & 0.0447 \\
\hline & MOWCA & 30.6576 & 0.0053 & 0.0167 & 0.0135 & 0.0641 & 0.0138 & 0.0651 & 0.1712 & 0.1756 & 0.2585 & 0.0540 \\
\hline \multirow{2}{*}{ Kursawe } & IMOWCA & 30.9350 & 0.0953 & 0.1146 & 0.1220 & 0.1265 & 0.0169 & 1.1172 & 1.2180 & 1.2587 & 1.2783 & 0.0879 \\
\hline & MOWCA & 34.8924 & 0.1359 & 0.1661 & 0.1536 & 0.1981 & 0.0291 & 0.8565 & 1.7162 & 1.5509 & 2.4648 & 0.6533 \\
\hline \multirow{2}{*}{ Viennet3 } & IMOWCA & 31.8706 & 0.0038 & 0.0057 & 0.0048 & 0.0082 & 0.0021 & 2.2716 & 2.5119 & 2.5766 & 2.7831 & 0.2106 \\
\hline & MOWCA & 30.6787 & 0.0046 & 0.0129 & 0.0114 & 0.0313 & 0.0064 & 2.0812 & 2.4289 & 2.4157 & 2.9181 & 0.2048 \\
\hline
\end{tabular}

28 Bold characters: the best results among all the algorithms. 
ZDT1

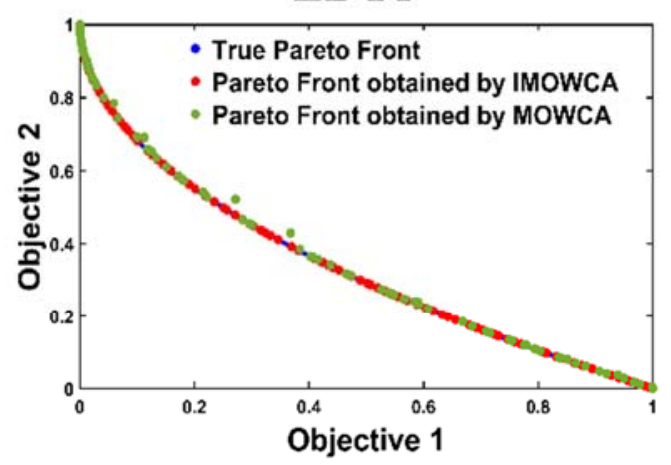

Kursawe

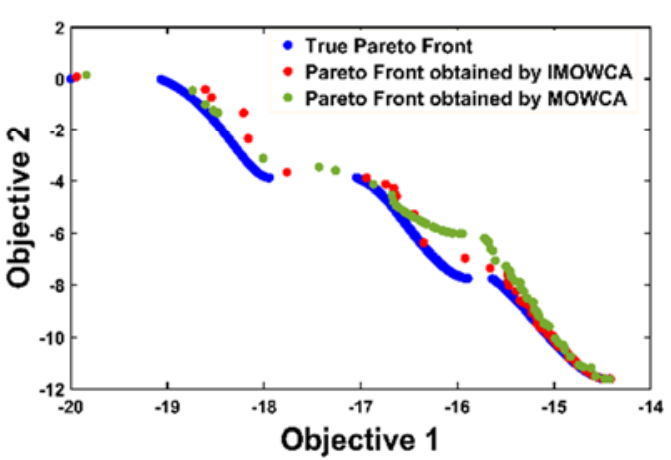

ZDT3

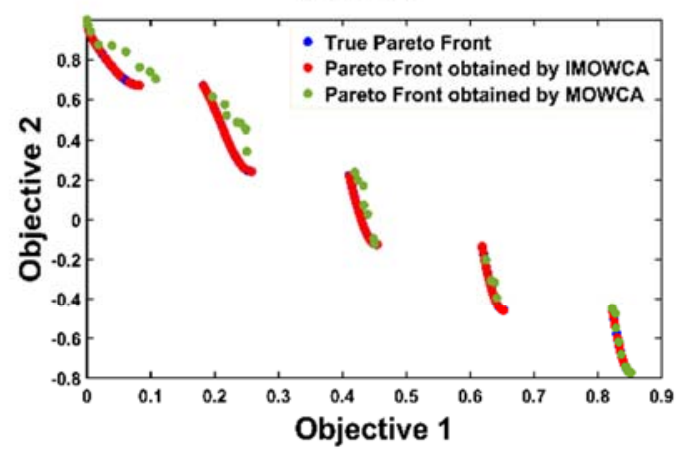

Viennet3

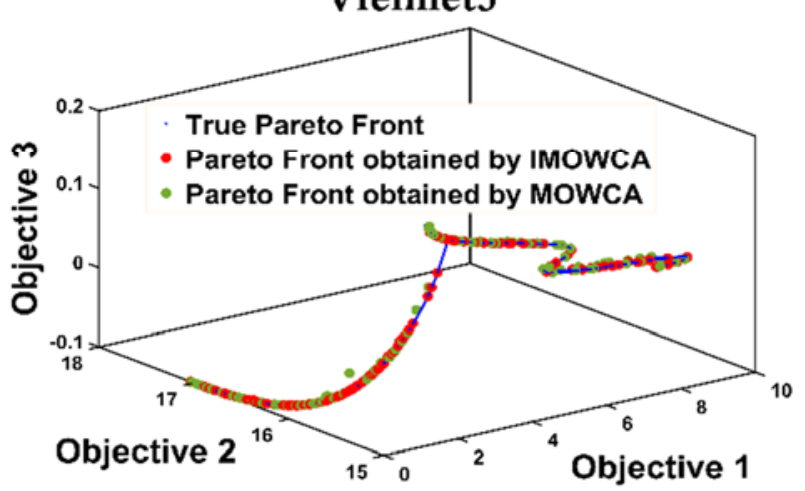

Fig. 2. Obtained Pareto fronts of improved multi-objective water cycle algorithm and the multi-objective water cycle algorithm.

\section{System Development}

In this section, the overall frame structure of the devised analysis-forecast system is systematicallydescribed, as well as the three popular metrics used for evaluating the performance of uncertainty modeling.

\subsection{System Design}

As shown in Fig. 3, the overall framework of the devised analysis-forecast system is composed of the followingsteps.

(1)In order to reduce the complexity generated bythe raw wind speed series, an effectivefrequency-time analysis based on the CEEMDAN method wasdevelopedto decompose the wind speed series into mode components.

(2)To analyze and explore the nonlinear dynamical mechanism of wind speed series and the corresponding IMFs, recurrence analysis techniques based on chaos theorywere developed to perform the qualitative and quantitative investigation for wind speed series.

(3)To ensurethe efficiency of the devised system, the IMFs generated fromthe original wind speed series are effectively merged according to the correspondingcomplexity degree.

(4)The C-C method based on chaostheory was developed to determine the optimal input forms of the forecast module according to the obtained delay time and embedding dimension, which improves the efficiency of the reconstruction of the model input.

(5)Furthermore, the input and output forms of the forecastmodule can be expressed as Eqs. (22) and (23), where $\boldsymbol{\alpha}$ denotes the interval width coefficient, andparameters $\tau$ and $m$ represent thedelay time and embedding dimension, respectively. 


Input set: $\left[\begin{array}{cc}x_{1} & x_{n-(m-1) \tau} \\ x_{1+\tau} & x_{n-(m-2) \tau} \\ x_{2+\tau} & x_{n-(m-2) \tau} \\ \mathrm{M} & \mathrm{M} \\ x_{1+(m-1) \tau} & x_{n-1}\end{array}\right](22)$
Output set: $\left[\begin{array}{cc}x_{1+(m-2) \tau} \times(1-\boldsymbol{\alpha}) & x_{1+(m-2) \tau} \times(1+\boldsymbol{\alpha}) \\ x_{2+(m-2) \tau} \times(1-\boldsymbol{\alpha}) & x_{2+(m-2) \tau} \times(1+\boldsymbol{\alpha}) \\ x_{3+(m-2) \tau} \times(1-\boldsymbol{\alpha}) & x_{3+(m-2) \tau} \times(1+\boldsymbol{\alpha}) \\ \mathrm{M} & \mathrm{M} \\ x_{n} \times(1-\boldsymbol{\alpha}) & x_{n} \times(1+\boldsymbol{\alpha})\end{array}\right]$ (23)

(6)More importantly, the IMOWCA proposed in this study was effectively developed to optimize the key parameters of MIMOLSSVM in the devised forecast module.

(7)Finally, the final prediction intervals can be obtained via merging the forecasting results generated by each IMF.
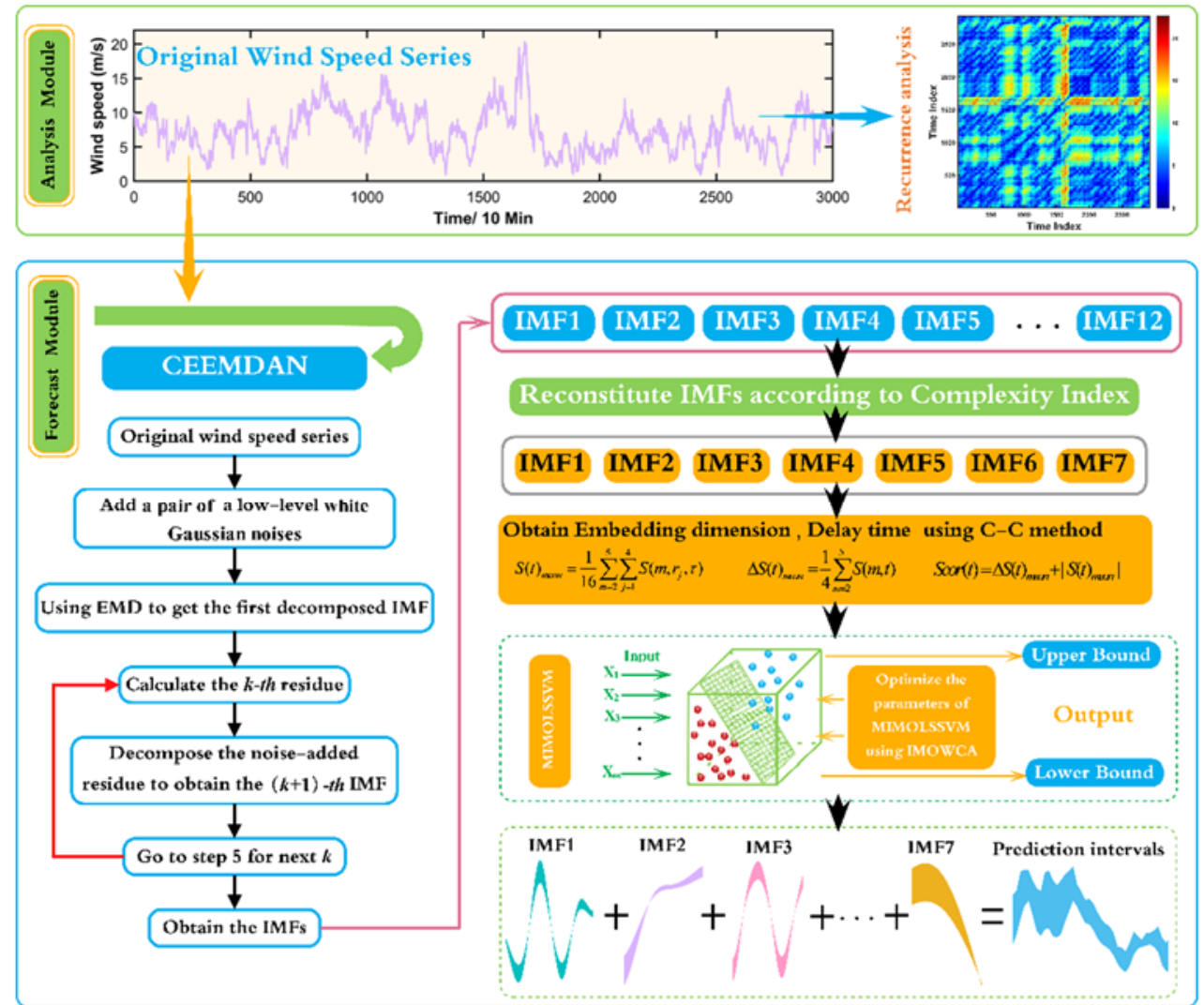

\subsection{System Evaluation}

In order to quantitativelyassess the effectiveness of the devised forecast module, the metrics coverage probability $(C P)$ and average width $(A W)$ were appliedin the evaluation module. Moreover, the accumulated width deviation $(A W D)$ metric was also usedto assess the reliability of theforecast module.

The accuracy of prediction intervals can be obtained by the $C P$ metric, which reflects the probability that the actual observed value $z_{i}$ falls within the constructed prediction interval. $C P$ can be calculated by 


$$
C P=\frac{1}{n} \sum_{i=1}^{n} c_{i}, \quad c_{i}=\left\{\begin{array}{cc}
1 & z_{i} \in\left[\boldsymbol{L}_{i}, \boldsymbol{U}_{i}\right] \\
0 & z_{i} \notin\left[\boldsymbol{L}_{i}, \boldsymbol{U}_{i}\right]
\end{array}\right.
$$

where $c_{i}$ signifies a Booleanvalue and $\boldsymbol{L}_{i}$ and $\boldsymbol{U}_{i}$ denote the lower and upper bound of theconstructed prediction interval, respectively. Parameter $n$ represents the number of prediction intervals.

Given the appropriate $C P$, the smaller the $A W$ value, the better isthe system performance is. The metric AW can be calculatedby

$$
A W=\frac{1}{n} \sum_{i=1}^{n}\left(\boldsymbol{U}_{i}-\boldsymbol{L}_{i}\right)
$$

$A W D$ can be calculated by measuring the relative deviation degree, which can be obtained by thecumulative sum of $A W D_{i}$. The calculation formula of $A W D$ isexpressed as Eqs. (26) and(27), where $\boldsymbol{\alpha}$ denotes the interval width coefficient and $\boldsymbol{I}_{i}$ represents the $i$-th prediction interval.

$$
\begin{gathered}
A W D_{i}=\left\{\begin{array}{cc}
\frac{\boldsymbol{L}_{i}^{(\alpha)}-z_{i}}{\boldsymbol{U}_{i}^{(\alpha)}-\boldsymbol{L}_{i}^{(\alpha)}}, & z_{i}<\boldsymbol{L}_{i}^{(\boldsymbol{\alpha})} \\
0, & z_{i} \in \boldsymbol{I}_{i}^{(\boldsymbol{\alpha})} \\
\frac{z_{i}-\boldsymbol{U}_{i}^{(\alpha)}}{\boldsymbol{U}_{i}^{(\alpha)}-\boldsymbol{L}_{i}^{(\alpha)}}, & z_{i}>\boldsymbol{U}_{i}^{(\boldsymbol{\alpha})}
\end{array}\right. \\
A W D^{(\alpha)}=\frac{1}{n} \sum_{i=1}^{n} A W D_{i}^{(\alpha)}(27)
\end{gathered}
$$

Importantly, it is worth mentioning that the metrics $C P$ and $A W$ were determined as the objective functions of IMOWCA in thisstudy.

\section{Numerical Simulations and Results Analysis}

In this section, the sites included in this study and the dataare described. Furthermore, certainstatistical metrics are used to express the basic characteristics ofwind speed series. In this study, recurrence analysis techniques were effectively developed to study the dynamic characteristics in phase space and uncover the rhythmicity of the nonlinear dynamics system based on wind speedseries. Finally, uncertainty modeling, which was effectively performed based on wind speed series from two wind farms, is described.

\subsection{Study Sites and Data Source}

In this section, wind speed series from two wind farms, namely, thePenglai site $\left(37.48^{\circ} \mathrm{N}, 120.45^{\circ} \mathrm{E}\right)$ and Chengde site $\left(40.97^{\circ} \mathrm{N}, 117.93^{\circ} \mathrm{E}\right)$ in China, were selected as the experimental data to verify the devised analysis-forecastsystem. As shown in Table 6, five statistical indexes Min, Max, Std. (standard deviation), complexity and maximum Lyapunov exponent (MLYE) based on thewolf method [85], were used to perform the descriptive statistical analysis of the dataused in this study. Theoretically, the studied nonlinear system can be assumed to be achaotic dynamic system if the maximum Lyapunov exponent is greater than zero.In particular, it is noteworthy that the MLYEs of the datain Table 6 are all greater than zero, which indicates that the wind speed series in this studyare essentiallychaotic time series. The basic information of the sites and the raw wind speed data, includingthe trainingand testing sets, are displayed in Fig. 4. 
Table 6. Statistical descriptions of the data.

\begin{tabular}{|c|c|c|c|c|c|c|c|}
\hline Sites & Data & Number & $\begin{array}{l}\text { Min } \\
(\mathrm{m} / \mathrm{s})\end{array}$ & $\begin{array}{l}\text { Max } \\
(\mathrm{m} / \mathrm{s})\end{array}$ & $\begin{array}{l}\text { Std. } \\
(\mathrm{m} / \mathrm{s})\end{array}$ & Complexity & MLYE \\
\hline \multirow{3}{*}{ Penglai site 1} & All samples & 3000 & 0.8 & 20.3 & 3.1379 & 0.3933 & 0.2432 \\
\hline & Training set & 2600 & 0.9 & 20.3 & 3.1994 & 0.3988 & 0.2555 \\
\hline & Testing set & 400 & 0.8 & 13.1 & 2.6134 & 0.4340 & 0.1525 \\
\hline \multirow{3}{*}{ Penglai site 2} & All samples & 3000 & 0.9 & 18.5 & 3.6152 & 0.3953 & 0.1592 \\
\hline & Training set & 2600 & 0.9 & 18.5 & 3.6791 & 0.4012 & 0.1534 \\
\hline & Testing set & 400 & 1.1 & 17.1 & 2.6444 & 0.5666 & 0.0417 \\
\hline \multirow{3}{*}{ Chengde site 1} & All samples & 3000 & 0.2 & 20.6 & 3.3785 & 0.5104 & 0.1818 \\
\hline & Training set & 2600 & 0.2 & 20.5 & 3.2912 & 0.4961 & 0.1564 \\
\hline & Testing set & 400 & 1.6 & 20.6 & 3.4708 & 0.6751 & 0.1972 \\
\hline
\end{tabular}

2

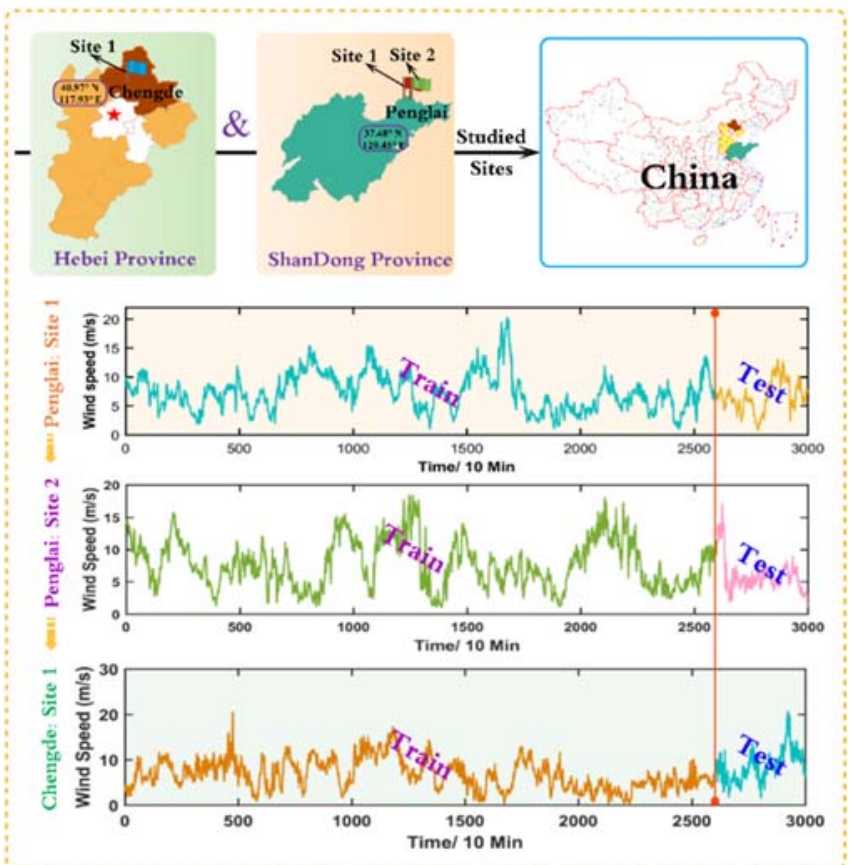

Fig. 4. Sites and data.

\subsection{Implementing Uncertainty Analysis and Modeling}

In this section, we describe howuncertainty modeling is effectively performed based on the nonlinear system of wind speed from two wind farms in China. Frequency domain decomposition based on theCEEMDAN technique is effectively applied to wind speed series.Then, the use of feature selection based on the C-C method to dynamically select the most qualified input forms is described.In addition, the development of the recurrence analysis techniques to explore the dynamic properties of wind speed series is presented. Finally, the effective simulation of the devised system to test its robustness and effectiveness is described.

\subsubsection{Frequency Domain Decomposition}

Because ofthe complex non-linearity of wind speed series, frequency domain decomposition for wind speed series is vital. CEEMDAN was developed in this studyto implementour method.It is noteworthy that no single theory can be used to effectively determine the number of IMFs far. In this study, the determination of the number of IMFs depended mainly on empirical study. Thedetailed parameters of CEEMDAN were as follows: the number of IMFs was 12; the standard deviation of the added Gaussian white noise was 0.2 ; the number of realizations was 500; and the maximum number of sifting iterations allowed was 5000. In order to reduce the 
1 modeling complexity and enhance the efficiency of the devised system, the IMFs 2 (IMF1-IMF12) generated by the CEEMDAN method were merged as shown in Fig. 35 , according to the corresponding complexity of each IMF, obtaining reconstructed 4 IMFs (IMF1-IMF7).The complexity degree and MLYE of these reconstructed IMFs 5 are displayed in Table 7. In Particular, it can be confirmedsubstantially that these 6 reconstructed IMFs are chaotic time series according to the MLYE in Table 7, which 7 are all greater than 0 .

8 Table 7.Complexity degree and of maximum Lyapunov exponent each intrinsic mode 9 function.

\begin{tabular}{|c|c|c|c|c|c|c|c|c|}
\hline Sites & Indexes & IMF1 & IMF2 & IMF3 & IMF4 & IMF5 & IMF6 & IMF7 \\
\hline \multirow{2}{*}{ Penglai site 1} & Complexity & 0.9455 & 0.7865 & 0.5794 & 0.4037 & 0.2573 & 0.1381 & 0.0397 \\
\hline & MLYE & 0.4457 & 0.0149 & 0.0573 & 0.0167 & 0.0079 & 0.0122 & 0.0054 \\
\hline \multirow{2}{*}{ Penglai site 2} & Complexity & 0.9350 & 0.7593 & 0.5878 & 0.4225 & 0.2677 & 0.1130 & 0.0523 \\
\hline & MLYE & 0.2874 & 0.0054 & 0.0102 & 0.0227 & 0.0347 & 0.0029 & 0.0066 \\
\hline \multirow{2}{*}{ Chengde site1 } & Complexity & 0.9141 & 0.7698 & 0.5962 & 0.4267 & 0.2740 & 0.1422 & 0.0439 \\
\hline & MLYE & 0.1167 & 0.0051 & 0.0068 & 0.0460 & 0.0050 & 0.0092 & 0.0147 \\
\hline
\end{tabular}

10

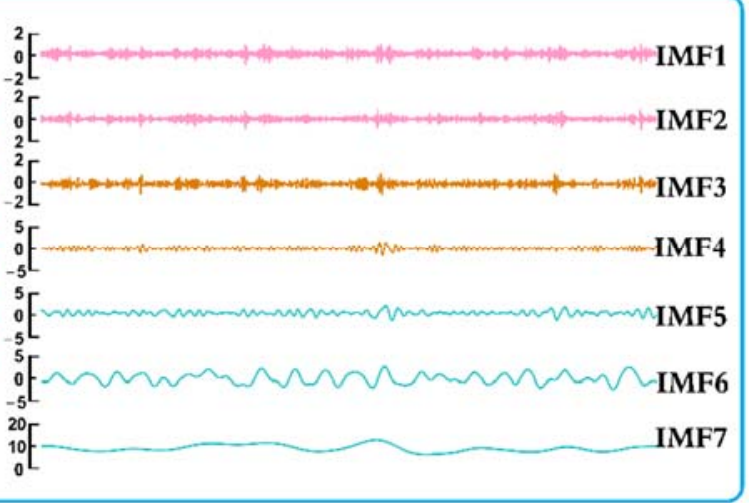

Fig. 5. Results of reconstructed intrinsic mode functions based on Penglai Site 1.

\subsubsection{Feature Selectionbased on Phase Space Reconstruction}

The determination of the suitable input forms forthe devised system plays a vital role in the process of uncertainty modeling. Inversely, inappositeinput forms will exert a significantlynegative impact on the forecast accuracy and effectiveness. Accordingly, the C-Cmethodbased on chaos theory was developed to dynamically determine the optimal input forms. Technically, the merits of the feature selection based on the C-C method are as follows: (1) model simplification [86]; (2) model efficiencyenhancement; (3) avoidance of the curse of dimensionality; and(4)model generalization enhancementby reducing over-fitting [87]. The C-Cmethodparameters of the wind speed seriesand corresponding IMFs are presented in Table 8. In order to effectively exhibit the attractor and its trajectories of wind speed series, the attractor of each IMF based on wind speed data from Penglaisite 1 was retrieved, according to the corresponding delay time obtained byusing theC-C method, as shown in Fig. 6. It can be seen in this figurethat the attractor is clearer and more unfolded from IMF1 to IMF7.The aforementioned analysis indicates that the predictability of IMF increases from IMF1 to IMF7. 
Table 8.Parameters $m, \tau$, and $\varpi$ generated by the C-C method.

\begin{tabular}{|c|c|c|c|c|c|c|c|c|}
\hline Indexes & Penglai site 1 & IMF1 & IMF2 & IMF3 & IMF4 & IMF5 & IMF6 & IMF7 \\
\hline$m$ & 3 & 5 & 17 & 6 & 10 & 8 & 4 & 5 \\
\hline$\tau$ & 37 & 10 & 2 & 4 & 8 & 16 & 31 & 33 \\
\hline$\varpi$ & 69 & 44 & 32 & 20 & 72 & 115 & 101 & 121 \\
\hline Indexes & Penglai site2 & IMF1 & IMF2 & IMF3 & IMF4 & IMF5 & IMF6 & IMF7 \\
\hline$m$ & 4 & 7 & 26 & 12 & 8 & 4 & 7 & 8 \\
\hline$\tau$ & 33 & 7 & 2 & 4 & 8 & 16 & 25 & 18 \\
\hline$\varpi$ & 93 & 45 & 50 & 45 & 55 & 53 & 141 & 121 \\
\hline Indexes & Chengde site1 & IMF1 & IMF2 & IMF3 & IMF4 & IMF5 & IMF6 & IMF7 \\
\hline$m$ & 5 & 9 & 48 & 19 & 6 & 10 & 5 & 7 \\
\hline$\tau$ & 30 & 5 & 2 & 4 & 8 & 15 & 31 & 21 \\
\hline$\varpi$ & 117 & 40 & 93 & 71 & 36 & 133 & 122 & 128 \\
\hline
\end{tabular}

2
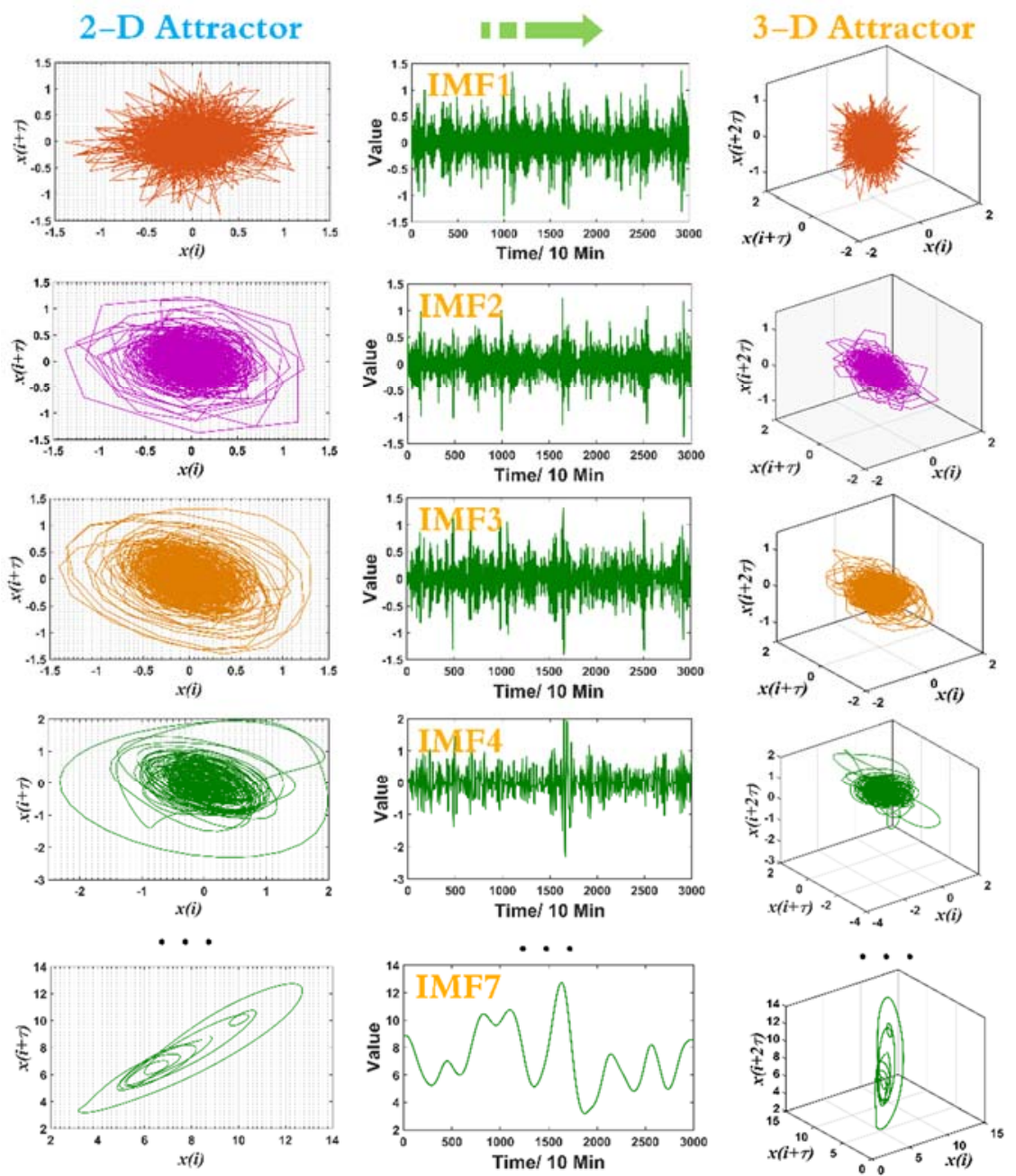

Fig. 6. Attractor of each intrinsic mode function based on Penglai site 1.

\subsubsection{Uncertainty Analysis}

The investigation and analysis of the dynamic characteristics and predictability in advance has animportant significance for uncertainty modeling. In order to qualitatively perform the analysis for the wind speed series, the original wind speed series and the corresponding IMF were transformed into recurrence plots according to the corresponding delay time and embedding dimension. Furthermore, the recurrence quantification analysis including certain metrics was used to quantitatively analyze and study the dynamic characteristics of the complex nonlinear system based on wind speed data. 
In order to effectively reconstruct the phase space, it is crucial to acquire the optimal delay time and embedding dimension. According to the delay times and embedding dimensions in Table 8, the original wind speed series and the corresponding IMFs could be transformed into recurrence plots, which are visualized in Fig. 7. Fig. 7(A), (B), and (C) show the data from Penglai site 1, Penglai site 2, and Chengde site 1 , respectively.

Fig. 7 indicates the following:

(1)Inthe recurrence plot of Penglai site 1 shown in Fig. 7(A), there are some short navy-blue diagonal lines, which simultaneously occur beside some single isolated points. The figure also further indicates that the wind speed series is chaotic. Additionally, the red bands signify that there is nonstationarity (or a drastic change). In Fig. 7-(A), a roughly homogeneous phenomenon appearsin therecurrence plots of IMF1-IMF4, which shows that a large number of single isolated points occur in these figures. The appearance of plentiful single isolated points and frequent red bands significantly illustrates that the series IMF1-IMF4 is extremely unstable, exposing the randomness and high volatility of wind speed series. The recurrence plots from IMF5 and IMF6 start to show some diagonal lines, which illustrates that the evolution of states in phase space is comparableat different times. There are some longer diagonal lines in the recurrence plots of IMF6 andIMF7, which indicates that the level of predictability is increasing gradually.

(2)It can be seen in the texture of therecurrence plot based on Penglaisite 2 inFig. 7-(B) that a homogeneous structure sometimes similarly occurs in the navy-blue squared block, which signifies that the stationary process is embedded into the nonlinear system based on wind speed series. Additionally, vertical recurrence points occur within the blocks, which indicates that it is achaos system among laminar zones. Similarly, there is anapproximately homogeneous texture among the recurrence plots of IMF1-IMF3, displaying manynavy-blue points with a uniform form, which further illustrates thatthe original wind speed data contain intrinsic random components. However, the navy-blue diagonal lines first appearin the recurrence plot of IMF4. Furthermore, there are longer diagonal lines in IMF6 andIMF7, in particularin IMF7. Additionally, some red clusters with vertical and horizontal textureappear, where anabrupt transition or change occurs.

(3)In Fig. 7-(C), the wind speed series and the corresponding IMFs from Chengde site 1 were converted into recurrence plots according to the corresponding delay time and embedding dimension. In the recurrence plot based on the wind speed series from Chengde site 1, there are very few navy-blue diagonal lines, which indicates there is strong indeterminacy and randomness in thewind speed data of this site. The indeterminacy and randomness makes uncertainty modeling challenging. As similar to Fig. 7-(A) and (B), IMF1-IMF3 also exhibit asimilar texture with manynavy-blue points, which means that these frequency domains have strong randomness components. Moreover, there are some periodic recurrent structures (the longer diagonal lines, checkerboard structures) in the recurrence plots of IMF4-IMF7, which indicates that the predictability of frequency domains is increasing gradually.

However, it is not sufficientto rely merely on subjective observation to investigate recurrence plots. Accordingly, in this study, evaluation metrics of therecurrence plots were usedto quantitatively evaluatethem.

Recurrence quantification analysis is an effective technique to investigate the phenomena of transitions, mutation, and periodicity inthe system dynamics in time series. Table 9shows some quantitative results of the aforementioned recurrence plots, which indicate the following. 
(1)Ahigher $R R, D E T$, and $L$ represent higher predictability. As compared to the wind speed data from Penglai sites 1-2, the predictability of the data fromtheChengde farm is lower according to the metrics consideredin this study. Additionally, the ENTR of wind speed data from Penglai site 2 is higher than fromPenglai site 1 and Chengde site 1 , which illustrates that the complexity of the recurrence plot based on the data from Penglai site 2 is higher than that for other sites.

(2)In all cases, in addition to the high frequency domains, namely, IMF1-IMF3, the four metrics increase from IMF4-IMF7, which significantly illustrates that IMF4-IMF7 generated by CEEMDAN are the main frequency domains of the studied wind speed series. However, merely modeling the main frequency does not sufficeto quantify the uncertainty. Accordingly, in this study, each IMF, including thehigh frequency domain and main frequency domain,was effectively modeled to further mine the uncertainty in wind speed series.

Remark: Given the evaluation metrics in Table 9, the wind speed series from Chengde site 1 has lower predictability because itsRR, DET, and Lare lower than those of Penglai sites 1-2. Accordingly, toeffectively perform uncertainty mining for Chengde site 1 is a challenging task. 

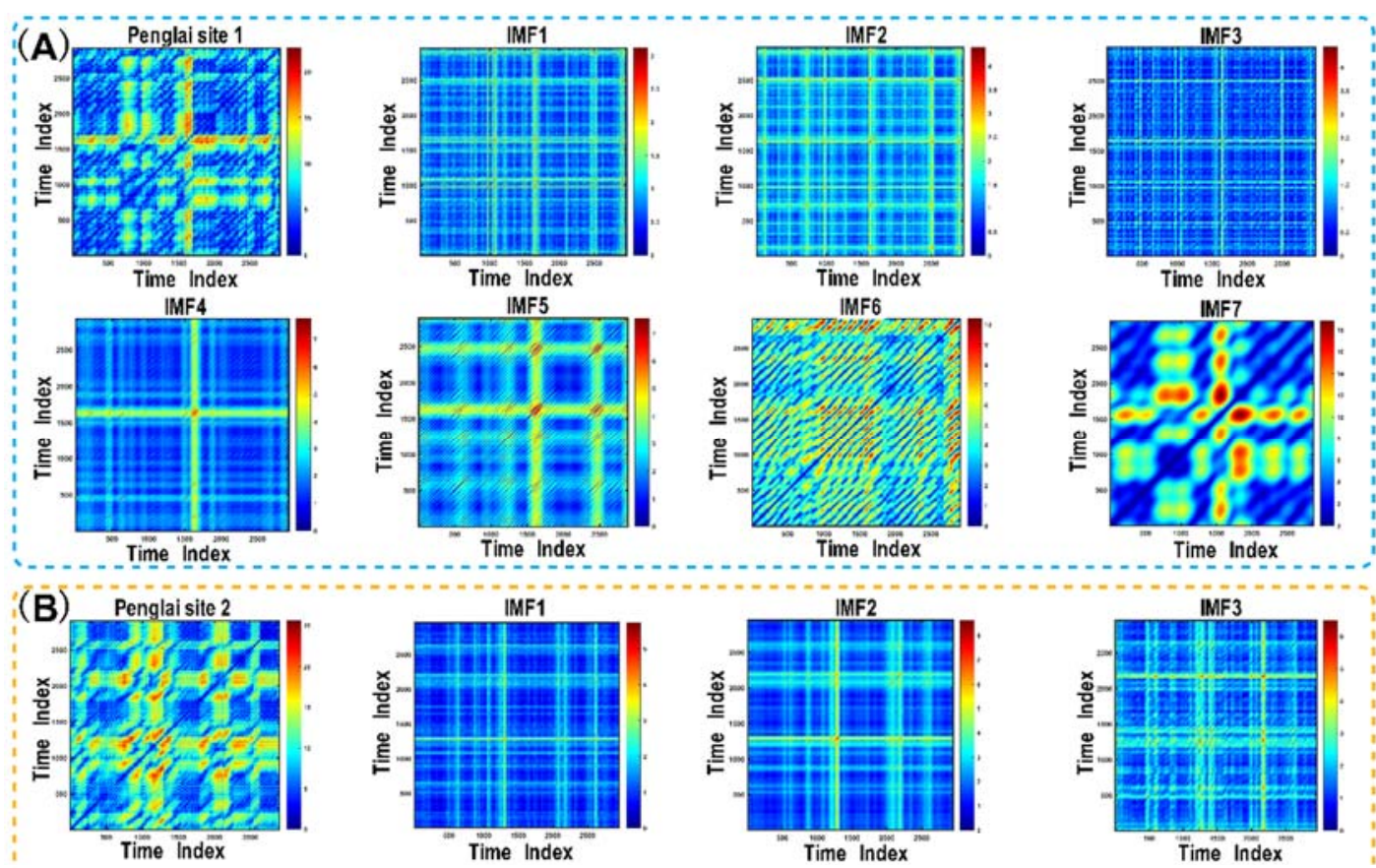

IMF3
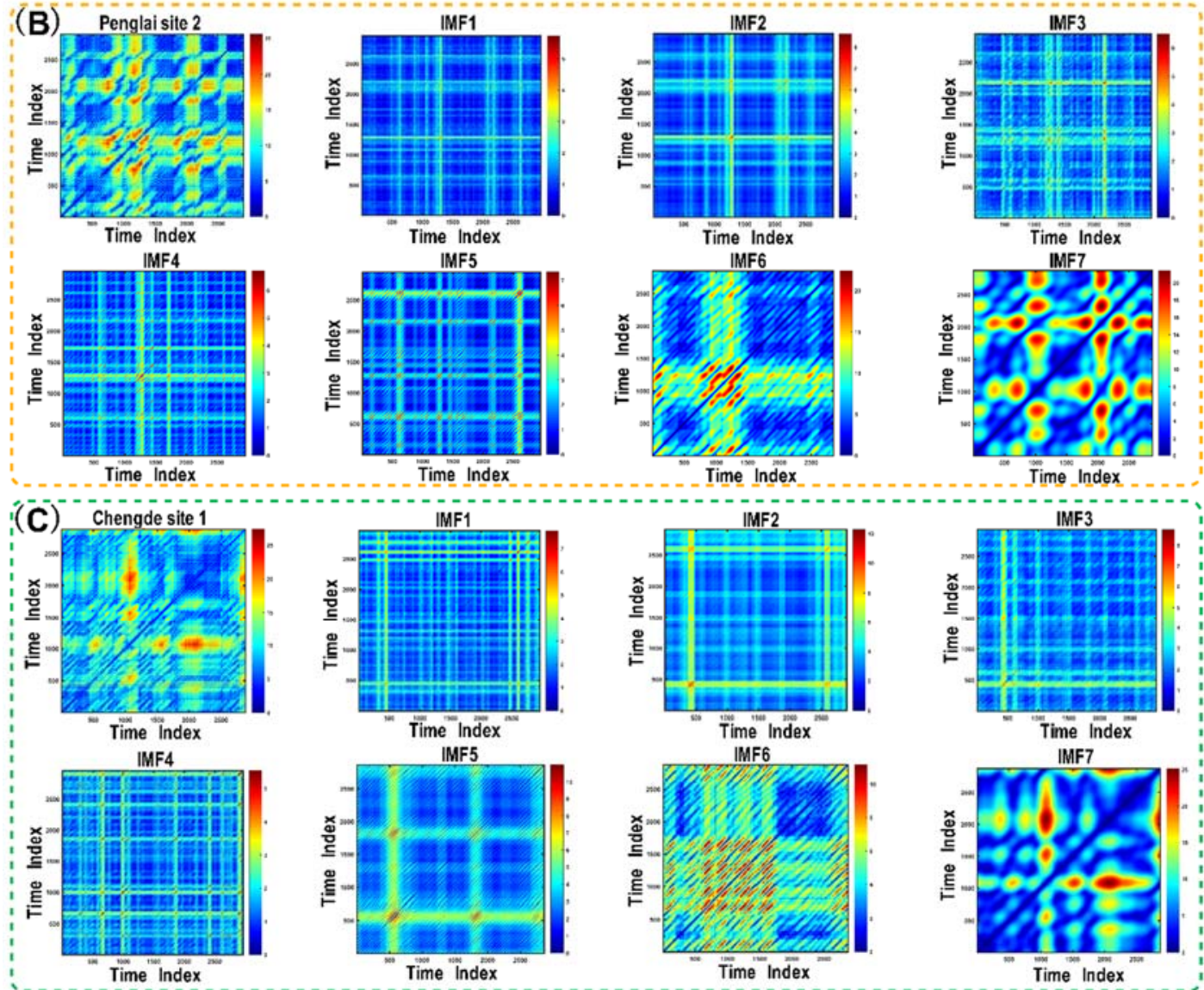

Fig. 7. Frequency-evolving recurrence plots of wind speed series.

3 Table 9.Results of recurrence quantification analysis based on mode components.

\begin{tabular}{lcccccccc}
\hline \multirow{2}{*}{ Indexes } & \multicolumn{7}{c}{ Penglaisite 1 } \\
\cline { 2 - 9 } & Original data & IMF1 & IMF2 & IMF3 & IMF4 & IMF5 & IMF6 & IMF7 \\
\hline Threshold & 1.41208 & 0.13248 & 0.11221 & 0.14394 & 0.20842 & 0.29988 & 0.71159 & 0.97515 \\
$\quad$ RR & 0.23898 & 0.00000 & 0.00000 & 0.00000 & 0.00000 & 0.00049 & 0.01493 & 0.13431 \\
DET & 0.93958 & 0.00000 & 0.00000 & 0.00000 & 0.00000 & 0.99853 & 0.99971 & 1.00000 \\
ENTR & 2.10174 & 0.00000 & 0.00000 & 0.00000 & 0.00000 & 2.81186 & 3.79254 & 5.84611 \\
$\quad L$ & 8.10338 & - & - & - & - & 23.36782 & 30.00095 & 192.73229 \\
\hline & & \multicolumn{7}{c}{ Penglai site 2 } \\
Threshold & 1.62684 & 0.16769 & 0.14856 & 0.17938 & 0.24270 & 0.32118 & 0.97036 & 0.98592 \\
RR & 0.22655 & 0.00000 & 0.00000 & 0.00000 & 0.00001 & 0.00242 & 0.02594 & 0.08798 \\
DET & 0.94656 & 0.00000 & 0.00000 & 0.00000 & 0.88462 & 0.94140 & 0.99995 & 0.99999 \\
ENTR & 2.15867 & 0.00000 & 0.00000 & 0.00000 & 1.01865 & 1.61122 & 4.63665 & 5.49362 \\
\hline
\end{tabular}




\begin{tabular}{lcccccccc}
\hline$L$ & 8.94314 & - & - & - & 2.87500 & 5.31190 & 58.46863 & 134.47020 \\
\hline & & & \multicolumn{7}{c}{ Chengde site 1 } \\
Threshold & 1.52034 & 0.24034 & 0.18110 & 0.18628 & 0.22991 & 0.35677 & 0.72102 & 1.08644 \\
RR & 0.08488 & 0.00000 & 0.00000 & 0.00000 & 0.00000 & 0.00290 & 0.00594 & 0.11729 \\
DET & 0.79644 & 0.00000 & 0.00000 & 0.00000 & 0.75000 & 0.94747 & 0.99992 & 0.99999 \\
ENTR & 1.26356 & 0.00000 & 0.00000 & 0.00000 & 0.64111 & 1.58960 & 3.71065 & 5.33671 \\
$L$ & 4.08779 & - & - & - & 2.25000 & 5.41086 & 66.42700 & 118.27968 \\
\hline
\end{tabular}

1

\subsubsection{Uncertainty Mining}

In this section, three cases based on wind speed series from two different wind farms in China are used to validate the effectiveness and robustness of the devised forecast module aimed at quantifying uncertainties. Three benchmark models, Modes-MOWCA-CC-MIMOLSSVM, IMOWCA-CC-MIMOLSSVM, and IMOWCA-MIMOLSSVM, were usedin this study in order to reveal the superiority of the devised system. Importantly, the crucial parameters of the forecast module were dynamically tuned by IMOWCA in order to ensure the robustness and accuracy of the system. The detailedparameter settings of IMOWCA are displayed in Table 10. Additionally, three statistical metrics, namely, $C P, A W$, and $A W D$, were applied to further evaluate the accuracy and appropriateness of the devised forecast module.

Table 10. Parameter settings of improved multi-objective water cycle algorithm.

\begin{tabular}{ccc}
\hline Parameter Configuration & & Default Value \\
${ } }$ & 4 \\
Population size & 50 \\
Range of population & & {$\left[e^{-5}, 1000\right]$} \\
Size of archive & 50 \\
Initial value of inertia weight & 0.9 \\
Terminal value of inertia weight & 0.45 \\
Number of streams & 46 \\
Maximumiteration number & 5 \\
Evaporation condition constant & $10^{-6}$ \\
Number of objectives & 2 \\
\hline
\end{tabular}

The quantitative simulation results of interval prediction are shown as in Tables 11-12, which consist of the simulations based on Penglaisite 1, Penglai site 2, and Chengde site 1, respectively. All the numerical simulations were conducted on the platform of MATLAB R2015b on MicrosoftWindows 7 with $3.30 \mathrm{GHz}$ Intel Core i5-4590HQ 64-bit and 8 GB of RAM. All thecases in this study were effectively implemented based on interval width coefficients 0.1 and 0.2 . To consider the randomness in the process of thesimulations, the obtained resultsshown in Tables 11-12 were determined via averaging the results of 10 experiments. Technically, the assessment of interval prediction is usually related to $C P$ and $A W$. However, there is a contradictory relationship between $C P$ and $A W$. Clearly, $C P$ increases when $A W$ increases, which reduces the informativeness of prediction intervals and increases risk. Accordingly, $A W D$ was introduced to effectivelyevaluate the accuracy of prediction intervals. Additionally, in order to illustrate the detailed prediction intervals of all the cases, Figs. 8-10 exhibit the performance of the devised forecast module and the benchmark modelsthat were considered, respectively.

In order to further investigate the experimentalresults, Tables 11-12and Figs. 8-10reveal the following.
(1)Acomparison
of 
Modes-MOWCA-CC-MIMOLSSVM reveals that,in all cases, the devised forecast module is notablysuperior to all thebenchmark models in generalaccording to $C P$, $A W$, and $A W D$, which signifies that IMOWCA has abetter capability to optimizethe devised system thanthe original MOWCA.For example, the average $C P$ value of the proposed Modes-IMOWCA-CC-MIMOLSSVM model reflects a 5.92\% and $1.75 \%$ improvement when the interval width coefficient is 0.1 and 0.2 , respectively,as compared to the benchmark model Modes-MOWCA-CC-MIMOLSSVM. Furthermore, the average $A W D$ value of the proposed model reflects a 0.0310 and 0.0081 improvementas compared to the benchmark model when the interval width coefficient is 0.1 and 0.2 , respectively.

(2)The proposal that mode components should be used to interval prediction in the devised system is a significant contribution of this study, since this is the first timemode components have beenapplied to the interval prediction of wind speed. As compared to the benchmark model IMOWCA-CC-MIMOLSSVM, which does not consider mode components, the comprehensive performance of the devised forecast module is superior to that ofthe other benchmark models, which illustrates the effectiveness and accuracy of the system.In summary, the experimental results in Tables 11-12 shows thatthe average $C P$ value of the proposed forecast system reflects a20.28\% and $8.40 \%$ improvementas compared to the benchmark model IMOWCA-CC-MIMOLSSVM when the interval width coefficient is 0.1 and 0.2 , respectively. Furthermore, the average $A W D$ value of the proposed forecast module reflects a 0.1120 and 0.0153 improvementas compared to the benchmark model when the interval coefficient is 0.1 and 0.2 , respectively.

(3)The C-Cmethodis an excellent meansof performing the feature selection in theforecastmodule, which can enhance the robustness and accuracy of the devised forecast module. As seen in Tables 11-12, the average $C P$ value of the proposed forecast module reflects a $21.24 \%$ and $9.97 \%$ improvementas compared to the benchmark model IMOWCA-MIMOLSSVM when the interval width coefficient is 0.1 and 0.2 , respectively. Furthermore, the average $A W D$ value of the proposed forecast module also reflects a 0.1350 improvementwhen theinterval width coefficient is 0.1 , and a 0.0294 improvementwhen the interval width coefficient is 0.2 as compared to the benchmark model. Accordingly, the effectiveapplication of theC-C methodto interval prediction of wind speed can be considered a contribution of this study.

(4)The performance and effectiveness of the system in the experiments based on Chengde site 1 are inferior to those based on the Penglai sites according to the metricsshown in Tables 11-12, which precisely verifies the remark in the section onuncertainty analysis.Consequently, it is necessary to perform uncertainty analysis of wind speed series, which can provide more information about their predictability, before the interval prediction of wind speed.

(5)Figs. 8-10 show that the prediction intervals yielded by the proposed forecast module are more accurate than those of the benchmark models, because the prediction intervals can cover true wind speed observations with a higher probability. Additionally, the constructed prediction intervals are smoother than those of the benchmark models, indicating that the robustness of the proposed prediction module is more stable.

(6)The results of the devised forecast module shows the same superiority when the experiments were conducted based on data from different wind farms, which further verifies the robustness and effectivenessof the devised system.

Remark: The results of the experiments based on the data fromdifferent wind 
1 farms significantly testifytothe advantages of the proposed forecast moduleon balance

2 ascompared to the benchmark models considered in this study.

$3 \quad$ Table 11.Testing results of system performance based on Penglai sites.

\begin{tabular}{|c|c|c|c|c|c|c|c|}
\hline Sites & \multicolumn{4}{|c|}{ Modes-IMOWCA-CC-MIMOLSSVM } & \multicolumn{3}{|c|}{ Modes-MOWCA-CC-MIMOLSSVM } \\
\hline \multirow{7}{*}{ Site 1} & $a$ & $A W$ & $C P$ & $A W D$ & $A W$ & $C P$ & $A W D$ \\
\hline & 0.1 & 1.4066 & $94.01 \%$ & 0.0193 & 1.4064 & $82.40 \%$ & 0.054 \\
\hline & 0.2 & 2.8001 & $96.25 \%$ & 0.0314 & 2.8230 & $98.88 \%$ & 0.0041 \\
\hline & \multicolumn{4}{|c|}{ IMOWCA-CC-MIMOLSSVM } & \multicolumn{3}{|c|}{ IMOWCA-MIMOLSSVM } \\
\hline & $\alpha$ & $A W$ & $C P$ & $A W D$ & $A W$ & $C P$ & $A W D$ \\
\hline & 0.1 & 1.3379 & $71.08 \%$ & 0.1361 & 1.3281 & $72.34 \%$ & 0.1066 \\
\hline & 0.2 & 2.6675 & $92 \%$ & 0.0210 & 2.6428 & $92.13 \%$ & 0.0341 \\
\hline \multirow{8}{*}{ Site 2} & \multicolumn{4}{|c|}{ Modes-IMOWCA-CC-MIMOLSSVM } & \multicolumn{3}{|c|}{ Modes-MOWCA-CC-MIMOLSSVM } \\
\hline & $\alpha$ & $A W$ & $C P$ & $A W D$ & $A W$ & $C P$ & $A W D$ \\
\hline & 0.1 & 0.9422 & $81.53 \%$ & 0.1202 & 1.1181 & $80.32 \%$ & 0.1753 \\
\hline & 0.2 & 2.1944 & $99.50 \%$ & 0.0013 & 2.1845 & $99.20 \%$ & 0.0014 \\
\hline & \multicolumn{4}{|c|}{ IMOWCA-CC-MIMOLSSVM } & \multicolumn{3}{|c|}{ IMOWCA-MIMOLSSVM } \\
\hline & $\alpha$ & $A W$ & $C P$ & $A W D$ & $A W$ & $C P$ & $A W D$ \\
\hline & 0.1 & 1.0904 & $69 \%$ & 0.1372 & 1.1824 & $66.24 \%$ & 0.1949 \\
\hline & 0.2 & 2.1770 & $89.67 \%$ & 0.0206 & 2.3530 & $86.55 \%$ & 0.0372 \\
\hline
\end{tabular}

4

Table 12. Testing results of system performance based on Chengde site 1 .

\begin{tabular}{|c|c|c|c|c|c|c|c|}
\hline Site & \multicolumn{4}{|c|}{ Modes-IMOWCA-CC-MIMOLSSVM } & \multicolumn{3}{|c|}{ Modes-MOWCA-CC-MIMOLSSVM } \\
\hline \multirow{7}{*}{ Site 1} & $\alpha$ & $A W$ & $C P$ & $A W D$ & $A W$ & $C P$ & $A W D$ \\
\hline & 0.1 & 1.9488 & $78.79 \%$ & 0.0656 & 1.9652 & $73.86 \%$ & 0.0689 \\
\hline & 0.2 & 3.9557 & $93.56 \%$ & 0.0229 & 3.9481 & $85.98 \%$ & 0.0744 \\
\hline & \multicolumn{4}{|c|}{ IMOWCA-CC-MIMOLSSVM } & \multicolumn{3}{|c|}{ IMOWCA-MIMOLSSVM } \\
\hline & $\alpha$ & $A W$ & $C P$ & $A W D$ & $A W$ & $C P$ & $A W D$ \\
\hline & 0.1 & 1.9122 & $53.41 \%$ & 0.2677 & 1.7203 & $52.03 \%$ & 0.3085 \\
\hline & 0.2 & 3.8426 & $82.44 \%$ & 0.0599 & 3.4371 & $80.71 \%$ & 0.0726 \\
\hline
\end{tabular}
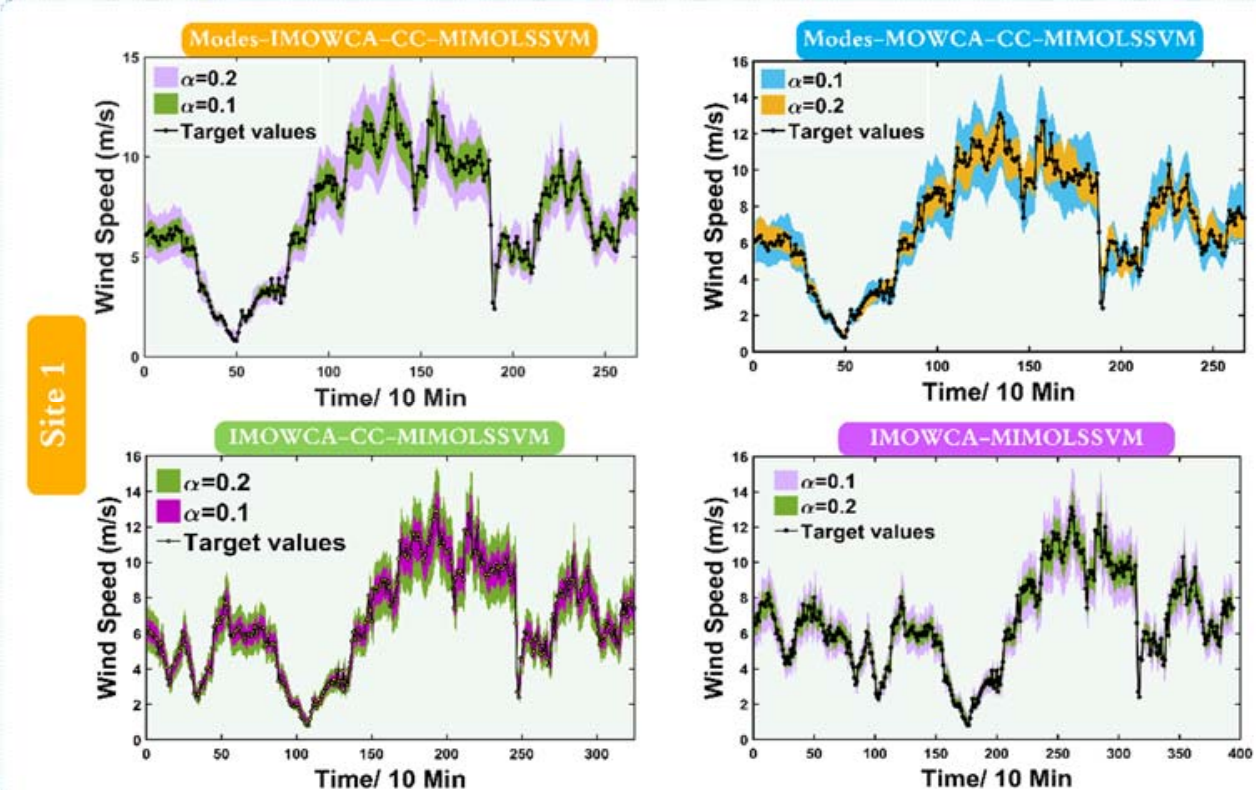

Fig. 8. Visualization of prediction intervals based on Penglai site 1. 

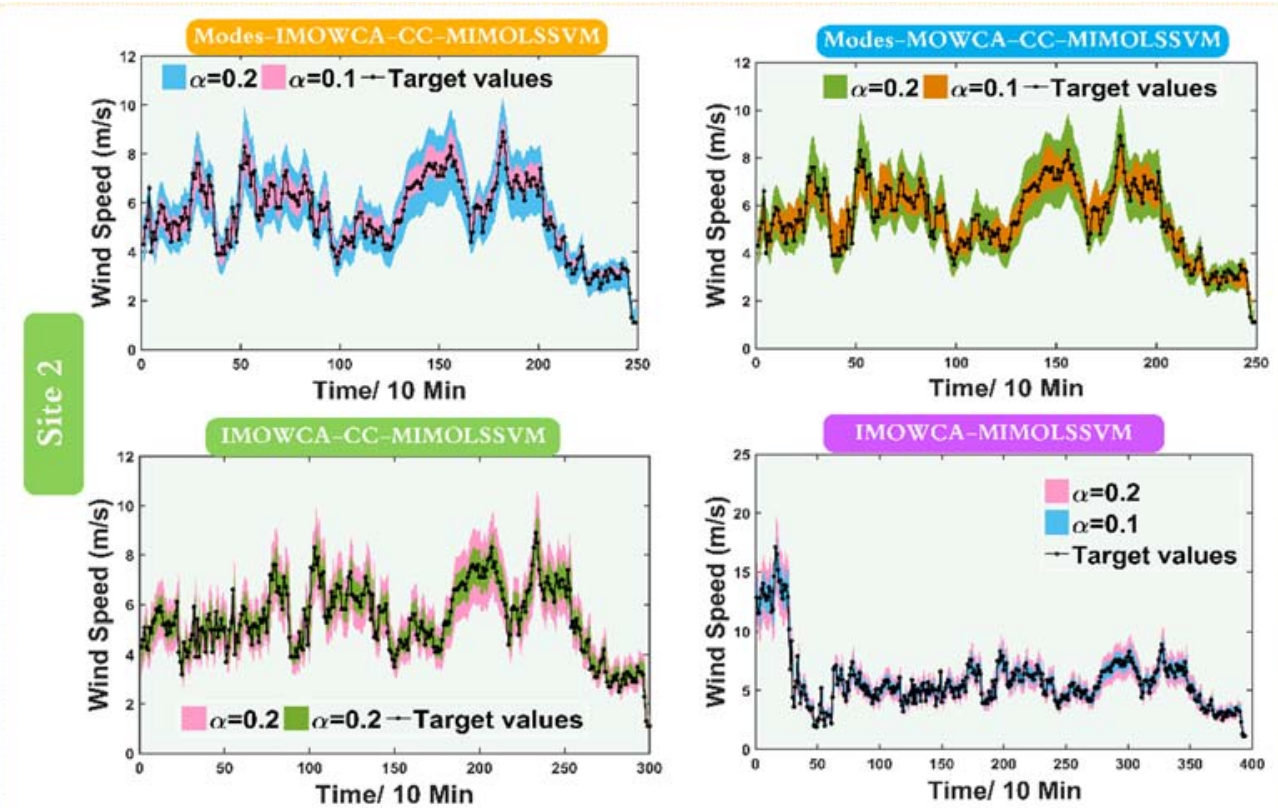
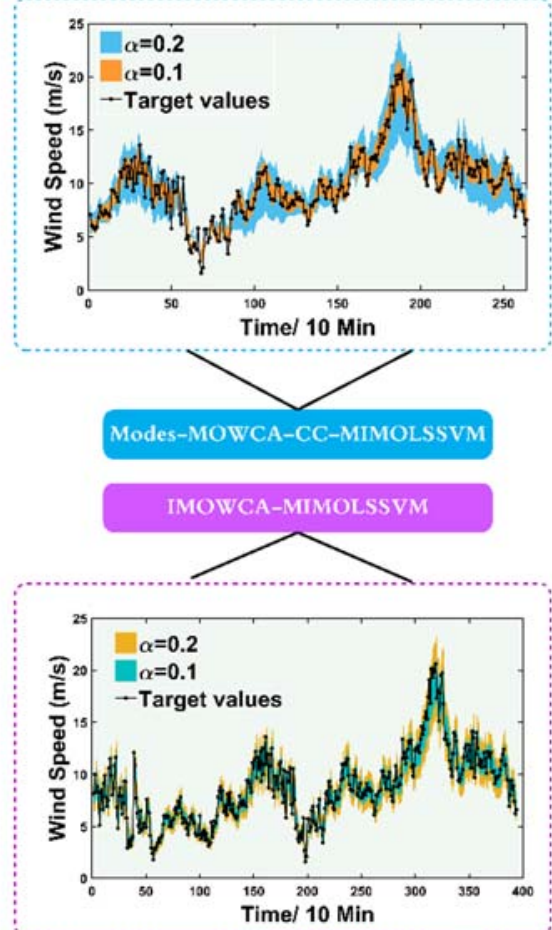

\section{Further Discussion}

Fig. 10.Visualization of prediction intervals based on Chengdesite 1.

In this section,the sensitivity analysis concerning iterations of IMOWCA is discussed based on different iteration numbers. Then, the practical significance and the applications of the proposed analysis-forecast system are also discussed. Finally, future research directions of interval prediction are suggested.

\subsection{Sensitivity Analysis on Iterations}

The iterations of amulti-objective optimization algorithm significantly affect the effectiveness and robustness of uncertainty modeling. An excessive number of iterations may yield over-fitting or fall into local optimum. Accordingly, the algorithm iterations of IMOWCA are discussed based on the wind speed series from Penglai site 
1. The simulation results are displayed in Table 13, from which the following conclusions can bedrawn.

(1)With anincrease in the number of iterations, the metric $A W$ shows a trend of fluctuations in different iterations, tending first to increase and then to decrease.The metric $C P$ displays a roughlydecreasing tendency, which also reveals that the accuracy and effectiveness of the devised model is declining. The metric $A W D$ showsatendency to increase with an increase initerations, which indicates that the average number offorecasting errors is increasing.

(2)In fact, thedifferent scenarios of uncertainty modeling rely largely onthe practical decision-making process. However, considering the performance and computational burden of the devised system, five can be considered a relatively optimal number of iterations on balance.

Table 13. Sensitivity analysis of different iterations based on improved multi-objective water cycle algorithm.

\begin{tabular}{ccccc}
\hline $\boldsymbol{\alpha}$ & Iteration & $\boldsymbol{A} \boldsymbol{W}$ & $\boldsymbol{C P}$ & $\boldsymbol{A} \boldsymbol{W D}$ \\
\hline & 5 & 1.4066 & $94.01 \%$ & 0.0193 \\
& 10 & 1.4144 & $86.89 \%$ & 0.1125 \\
0.1 & 30 & 1.4052 & $88.39 \%$ & 0.0185 \\
& 60 & 1.4289 & $49.44 \%$ & 2.6442 \\
& 100 & 1.4295 & $19.85 \%$ & 5.2260 \\
& 150 & 1.3802 & $46.44 \%$ & 0.8631 \\
& 200 & 1.3678 & $72.28 \%$ & 0.6253 \\
\hline \multirow{4}{*}{0.2} & 5 & 2.8001 & $96.25 \%$ & 0.0314 \\
& 10 & 2.8279 & $96.15 \%$ & 0.0031 \\
& 30 & 2.7265 & $71.91 \%$ & 0.2891 \\
& 60 & 2.7211 & $73.03 \%$ & 3.2407 \\
& 100 & 2.8226 & $44.94 \%$ & 0.5238 \\
& 150 & 2.7060 & $64.79 \%$ & 0.2250 \\
& 200 & 2.7357 & $72.28 \%$ & 0.6268 \\
\hline
\end{tabular}

\subsection{Practical Significance and Implications of the Proposed System}

The results of forecasting wind speed, especially of point forecasting, will inevitably produce some bias because of the high randomness of wind speed, which will have a negative influence on the robust scheduling and management of wind power systems in a wind farm. However, effective interval prediction is conducive to mitigating this negative influence. The amount of wind power generated is directly dependent on the wind speed; the formula for the conversion of wind speed to wind power is provided in [88]. In general, effective and comprehensive wind speed forecasting, which plays a major role in maintaining the stability of the wind power system further [52] and improving the efficiency of wind power generation, is urgently needed. Currently, most wind farms focus mainly on point forecasting. However, the investigation and application of interval prediction for wind speed has not received major attention.

As a complement to the current wind power system, the proposed wind speed analysis-forecast system, aimed at providing effective prediction intervals, has great potential to be integrated into the data platform in a wind farm to allow better operation and scheduling of the wind power systems.

Additionally, accurate wind speed forecasting is needed in an effective assessment of wind power, because wind energy is directly proportional to the cube of wind speed and the assessment of potential wind capacity depends ultimately on robust wind speed forecasting [89]. 
Although the proposed analysis-forecast system shows a good performance in the uncertainty modeling of wind speed, there remain aspects of this system that need further improvement, which can be summarized as follows.

(1) The proposed analysis-forecast system is focused mainly on short-term interval prediction of wind speed. More effort can be invested in long-term interval prediction of wind speed to further improve the efficiency of operation and scheduling in a wind power system.

(2) In the preprocessing module of the analysis-forecast system, CEEMDAN was developed to refine the wind speed series. However, thus far no perfect theory exists that can help effectively determine the number of IMFs when using CEEMDAN. In the system, the number of IMFs in CEEMDAN was determined by empirical studies. Accordingly, the effective determination of the appropriate number of IMFs when developing wind speed forecasting should be investigated in future studies.

\subsection{Future Scope}

In this study, a comprehensive analysis-forecast system including uncertainty analysis and mining of wind speed was developed. In order to effectively quantify the uncertainty existing in a nonlinear system based on wind speed, the development of new orientations aimed at uncertainty analysis and mining is verynecessary. More extensive explorations and investigations in the field should be conducted. Someresearch directions that should be considered are as follows.

(1)Technically, the physical models based on numerical weather prediction have an advantage in long-term forecasting. Accordingly, combining the physics models and statistical models to perform uncertainty modeling is a not only worthwhile, but also promising direction.

(2)The evaluation metrics of interval prediction should be further investigated in extensive research studies in order to allow a more effective evaluation of the interval prediction models.

(3)In recent years, dynamic multi-objective optimization algorithms have been receiving considerableattention inextensive studiesbecause of theirexcellent capability for solvingdynamic optimization problems. The uncertainty modeling of wind speedin practice usually involves adynamic and complex environment. Accordingly, the application of dynamic multi-objective optimization algorithms to the field of wind speed or wind power forecasting appears to be a promising research direction.

(4)Deep learning models, as an emerging technology, have been applied to many fields recently. However, the development and application of deep learning techniques to perform uncertainty modeling has rarely received attention, and thus, constitutes a promising research direction.

\section{Conclusions}

With the exhaustion of traditional energy, wind energy is consistently being evaluated worldwide as a promising alternative because of its sustainability and cleanness.However, thefurther development of wind energy is significantly restricted because ofits inherent intermittency and randomness, which possibly put the operation and scheduling of wind farms at risk. In order to more effectively analyze and mine the uncertainty of wind speed, recurrence analysis based on chaos techniqueswasdeveloped in this studyto reveal the inherent dynamic characteristics of wind speed, which is vital for exploring the predictability and modelingofthe uncertainty of wind speed. Furthermore, an effective forecast module integrating mode components, chaos techniques, and IMOWCAwas successfully devised. Importantly, mode components (or frequency domains) were developed for the first time to perform uncertainty modeling, which was proved to be significantly 
1 moreeffective and robust thanthe benchmark models consideredin this study. 2 Furthermore, MOWCA was further developed by introducing the adaptive inertia 3 weight, leading to a novel multi-objective algorithm, namely, IMOWCA. The results 4 ofnumerical experiments to test the algorithm clearly illustrate that IMOWCA is a 5 significant improvementonthe original MOWCA on balance.Finally, extensive 6 experiments usingquantitative metrics revealedthe significant effectiveness and 7 superiority of the system in this study. Additionally, given the excellent performance 8 of the devised system, it can also be applied in practicein the fields of load forecasting, 9 wind power forecasting, and stock forecasting, and so forth.

\section{Conflict of interests}

11 The authors declare that there is no conflict of interests regarding the publication of 12 this paper.

\section{Acknowledgements}

14 This research was supported by the National Natural Science Foundation of China 15 (Grant No. 71671029 and Grant No. 41475013).

\begin{tabular}{|c|c|c|c|}
\hline \multicolumn{4}{|c|}{ Nomenclature } \\
\hline WPI & wind power integration & $\tau$ & delay time \\
\hline WSFM & wind speed forecast model & $m$ & embedding dimension \\
\hline WSF & wind speed forecast & $\varpi$ & time window \\
\hline AR & autoregressive model & $\|\cdot\|$ & a norm \\
\hline ARIMA & $\begin{array}{l}\text { autoregressive integrated moving average } \\
\text { model }\end{array}$ & diag & diagonal matrix \\
\hline $\mathrm{ARCH}$ & $\begin{array}{l}\text { autoregressive conditional } \\
\text { heteroskedasticitymodel }\end{array}$ & LB & the lower bound of variables \\
\hline ANNs & artificial neural networks & UB & the upper bound of variables \\
\hline PSO & particle swarm optimization & max iteration & the maximum iteration number \\
\hline GA & genetic algorithm & $\omega^{-}$ & adaptive inertia weight \\
\hline LUBE & lower upper bound estimation & GD & generational distance \\
\hline ELM & extreme learning machine & SP & spacing \\
\hline LLFNN & local linear fuzzy neural network & $C P$ & coverage probability \\
\hline RBFNN & radial basis function neural network & $A W$ & average width \\
\hline WNN & wavelet neural network & $A W D$ & accumulated width deviation \\
\hline MIMO-LSSVM & $\begin{array}{l}\text { multi-input multi-output least squares } \\
\text { support vector machine }\end{array}$ & $\boldsymbol{L}_{i}$ & $\begin{array}{l}\text { lower bound of } i \text {-th prediction } \\
\text { interval }\end{array}$ \\
\hline WCA & water cycle algorithm & $\boldsymbol{U}_{i}$ & $\begin{array}{l}\text { upper bound of } i \text {-th prediction } \\
\text { interval }\end{array}$ \\
\hline IMOWCA & $\begin{array}{l}\text { Improved multi-objective water cycle } \\
\text { algorithm }\end{array}$ & $c_{i}$ & a Boolean value \\
\hline EMD & empirical mode decomposition & $\varsigma$ & $\begin{array}{l}\text { predefined threshold in recurrence } \\
\text { analysis }\end{array}$ \\
\hline EEMD & ensemble empirical mode decomposition & $P(l)$ & $\begin{array}{l}\text { the probability to find a diagonal } \\
\text { line of length } l \text { inthe recurrence plot. }\end{array}$ \\
\hline CEEMD & $\begin{array}{l}\text { complete ensemble empirical mode } \\
\text { decomposition }\end{array}$ & $\Phi(\cdot) / \phi(\cdot)$ & the nonlinear mapping \\
\hline CEEMDAN & $\begin{array}{l}\text { complete ensemble empirical mode } \\
\text { decomposition with adaptive noise }\end{array}$ & $\alpha$ & interval width coefficient \\
\hline IMFs & intrinsic mode functions & $\boldsymbol{I}_{i}$ & the $i$-th prediction interval \\
\hline MIMO-LSSVM & $\begin{array}{l}\text { multi-input multi-output least squares } \\
\text { support vector machine }\end{array}$ & rand & $\begin{array}{l}\text { a uniformly distributed random } \\
\text { number in }[0,1]\end{array}$ \\
\hline WCA & water cycle algorithm & $N_{s r}$ & the number of streams \\
\hline$R R$ & recurrence rate & $N_{\text {pop }}$ & the number of raindrops \\
\hline$D E T$ & determinism & $d_{\max }$ & a small number close to zero \\
\hline ENTR & entropy & $\Theta(\cdot)$ & Heavisible function \\
\hline$L$ & average diagonal line length & Cost $_{n}$ & the fitness value of the $n$-thraindrop \\
\hline $\overrightarrow{\mathbf{X}}_{\text {River }}^{i}$ & the position of River & $T$ & training dataset \\
\hline
\end{tabular}




\begin{tabular}{|llll|}
\hline $\overrightarrow{\mathbf{X}}_{\text {Sea }}^{i}$ & The position of sea & MLYE & maximum lyapunov exponent \\
$R R$ & recurrence rate & $d_{\max }$ & the tolerance in IMOWCA \\
$D E T$ & determinism & $S(t)_{\text {mean }}$ & standard deviation \\
ENTR & entropy & $\Delta S(t)_{\text {mean }}$ & a statistic shown in Eq. (7) \\
$\overrightarrow{\mathbf{X}}_{\text {Stream }}^{i}$ & The position of stream & a statistic shown in Eq. (8) \\
Randn & an uniformly distributed random numbers & $S c o r(t)$ & a statistic shown in Eq. (9) \\
$C$ & in [1,1] & $\square^{p}$ & input space with the dimension of $p$ \\
$z_{i}$ & a constant in Eqs. (13-15) \& (21) & MLYE & maximum lyapunov exponent \\
\hline
\end{tabular}

1

2 


\section{Appendix}

Multi-objective test functions used in this paper.

Table A. Testing problems.

\begin{tabular}{|c|c|c|c|c|c|c|}
\hline Problem & Dimension & Range & & Expression & Continuity & Convexity \\
\hline ZDT1 & 30 & {$[0,1]$} & Minimize $=$ & $\left\{\begin{array}{l}f_{1}(x)=x_{1} \\
f_{2}(x)=g(x) \times \boldsymbol{h}\left(f_{1}(x), g(x)\right) \\
g(x)=1+\frac{9}{29} \sum_{i=2}^{30} x_{i} \\
\boldsymbol{h}\left(f_{1}(x), g(x)\right)=1-\sqrt{\frac{f_{1}(x)}{g(x)}}\end{array}\right.$ & $\checkmark$ & $\checkmark$ \\
\hline ZDT3 & 30 & {$[0,1]$} & Minimize $=$ & $\left\{\begin{array}{l}f_{1}(x)=x_{1} \\
f_{2}(x)=g(x) \times \boldsymbol{h}\left(f_{1}(x), g(x)\right) \\
g(x)=1+\frac{9}{29} \sum_{i=2}^{30} x_{i} \\
\boldsymbol{h}\left(f_{1}(x), g(x)\right)=1-\sqrt{\frac{f_{1}(x)}{g(x)}}-\left(\frac{f_{1}(x)}{g(x)}\right) \times \sin \left(10 \pi f_{1}(x)\right)\end{array}\right.$ & $x$ & $\checkmark$ \\
\hline Kursawe & 3 & {$[-5,5]$} & Minimize $=$ & $\left\{\begin{array}{l}f_{1}(x)=\sum_{i=1}^{2}\left[-10 \exp \left(-0.2 \sqrt{x_{i}^{2}+x_{i+1}^{2}}\right)\right] \\
f_{2}(x)=\sum_{i=1}^{3}\left[\left|x_{i}\right|^{0.8}+5 \sin \left(x_{i}^{3}\right)\right]\end{array}\right.$ & $x$ & $x$ \\
\hline Viennet3 & 2 & {$[-3,3]$} & Minimize $=$ & $\left\{\begin{array}{l}f_{1}(x, y)=0.5\left(x^{2}+y^{2}\right)+\sin \left(x^{2}+y^{2}\right) \\
f_{2}(x, y)=\frac{(3 x-2 y+4)^{2}}{8}+\frac{(x-y+1)^{2}}{27}+15 \\
f_{3}(x, y)=\frac{1}{\left(x^{2}+y^{2}+1\right)}-1.1 \exp \left(-x^{2}-y^{2}\right)\end{array}\right.$ & $\checkmark$ & - \\
\hline
\end{tabular}




\section{References}

[1] Pourbeik P, Akhmatov V, Akiyama Y, et al. Modelling and Dynamic Behavior of Wind Generation as it Relates to Power System Control and Dynamic Performance[J]. 2007.

[2] Yu J, Ji F, Zhang L, Chen Y. An over painted oriental arts: Evaluation of the development of the Chinese renewable energy market using the wind power market as a model. Energy Policy 2009;37:5221-5. doi:10.1016/j.enpol.2009.07.035.

[3] Wang Y, Wang J, Wei X. A hybrid wind speed forecasting model based on phase space reconstruction theory and Markov model: A case study of wind farms in northwest China. Energy 2015;91:556 - 72. doi:10.1016/j.energy.2015.08.039.

[4] Baseer MA, Meyer JP, Rehman S, Mahbub AM, Al-Hadhrami LM, Lashin A. Performance evaluation of cup-anemometers and wind speed characteristics analysis. Renew Energy 2016;86:733-44. doi:10.1016/j.renene.2015.08.062.

[5] Santamaría-Bonfil G, Reyes-Ballesteros A, Gershenson C. Wind speed forecasting for wind farms: A method based on support vector regression. Renew Energy 2016;85:790-809. doi:10.1016/j.renene.2015.07.004.

[6] Wang J, Qin S, Zhou Q, Jiang H. Medium-term wind speeds forecasting utilizing hybrid models for three different sites in Xinjiang, China. Renew Energy 2015;76:91 - 101. doi:10.1016/j.renene.2014.11.011.

[7] Hu Q, Zhang R, Zhou Y. Transfer learning for short-term wind speed prediction with deep neural networks. Renew Energy 2016;85:83-95. doi:10.1016/j.renene.2015.06.034.

[8] Telesca L, Lovallo M, Kanevski M. Power spectrum and multifractaldetrended fluctuation analysis of high-frequency wind measurements in mountainous regions. Appl Energy 2016;162:1052-61. doi:10.1016/j.apenergy.2015.10.187.

[9] Bigdeli N, Afshar K, Gazafroudi AS, Ramandi MY. A comparative study of optimal hybrid methods for wind power prediction in wind farm of Alberta, Canada. Renew Sustain Energy Rev 2013;27:20-9. doi:10.1016/j.rser.2013.06.022.

[10] Yu YSW, Sun D, Zhang J, Xu Y, Qi Y. Study on a Pi-type mean flow acoustic engine capable of wind energy harvesting using a CFD model. Appl Energy 2017;189:602-12. doi:10.1016/j.apenergy.2016.12.022.

[11] Sun D, Xu Y, Chen H, Wu K, Liu K, Yu Y. A mean flow acoustic engine capable of wind energy harvesting. Energy Convers. Manag., vol. 63, 2012, p. 101-5. doi:10.1016/j.enconman.2011.12.035.

[12] Yu Y, Sun D, Wu K, Xu Y, Chen H, Zhang X, et al. CFD study on mean flow engine for wind power exploitation. Energy Convers Manag 2011;52:2355-9. doi:10.1016/j.enconman.2010.12.046.

[13] Jung J, Broadwater RP. Current status and future advances for wind speed and power forecasting. Renew Sustain Energy Rev 2014;31:762-77. doi:10.1016/j.rser.2013.12.054.

[14] Zhao J, Guo Y, Xiao X, Wang J, Chi D, Guo Z. Multi-step wind speed and power forecasts based on a WRF simulation and an optimized association method. Appl Energy 2017;197:183-202. doi:10.1016/j.apenergy.2017.04.017.

[15] Lei M, Shiyan L, Chuanwen J, Hongling L, Yan Z. A review on the forecasting of wind speed and generated power. Renew Sustain Energy Rev 2009;13:915-20. doi:10.1016/j.rser.2008.02.002.

[16] Costa A, Crespo A, Navarro J, Lizcano G, Madsen H, Feitosa E. A review on the young history of the wind power short-term prediction. Renew Sustain Energy Rev 2008;12:1725-44. doi:10.1016/j.rser.2007.01.015. 
[17]Landberg L, Giebel G, Nielsen HA, Nielsen T, Madsen H. Short-term prediction An overview. Wind Energy 2003;6:273-80. doi:10.1002/we.96.

[18] Cassola F, Burlando M. Wind speed and wind energy forecast through Kalman filtering of Numerical Weather Prediction model output. Appl Energy 2012;99:154-66. doi:10.1016/j.apenergy.2012.03.054.

[19] Jung J, Broadwater RP. Current status and future advances for wind speed and power forecasting. Renew Sustain Energy Rev 2014;31:762-77. doi:10.1016/j.rser.2013.12.054.

[20] Poggi P, Muselli M, Notton G, Cristofari C, Louche A. Forecasting and simulating wind speed in Corsica by using an autoregressive model. Energy Convers Manag 2003;44:3177-96. doi:10.1016/S0196-8904(03)00108-0.

[21] Erdem E, Shi J. ARMA based approaches for forecasting the tuple of wind speed and direction. Appl Energy 2011;88:1405-14. doi:10.1016/j.apenergy.2010.10.031.

[22] Torres JL, García A, De Blas M, De Francisco A. Forecast of hourly average wind speed with ARMA models in Navarre (Spain). Sol Energy 2005;79:65-77. doi:10.1016/j.solener.2004.09.013.

[23] Erdem E, Shi J. ARMA based approaches for forecasting the tuple of wind speed and direction. Appl Energy 2011;88:1405-14. doi:10.1016/j.apenergy.2010.10.031.

[24] Kavasseri RG, Seetharaman K. Day-ahead wind speed forecasting using f-ARIMA models. Renew Energy 2009;34:1388 - 93. doi:10.1016/j.renene.2008.09.006.

[25] Wang M Di, Qiu QR, Cui BW. Short-term wind speed forecasting combined time series method and arch model. Proc. - Int. Conf. Mach. Learn. Cybern., vol. 3, 2012, p. 924 - 7. doi:10.1109/ICMLC.2012.6359477.

[26] Cadenas E, Rivera W. Short term wind speed forecasting in La Venta, Oaxaca, Mexico, using artificial neural networks. Renew Energy 2009;34:274-8.

[27] Guo Z, Wu J, Lu H, Wang J. A case study on a hybrid wind speed forecasting method using BP neural network. Knowledge-Based Syst 2011;24:1048-56. doi:10.1016/j.knosys.2011.04.019.

[28] Monfared M, Rastegar H, Kojabadi HM. A new strategy for wind speed forecasting using artificial intelligent methods. Renew Energy 2009;34:845-8. doi:10.1016/j.renene.2008.04.017.

[29] Guo Z, Zhao W, Lu H, Wang J. Multi-step forecasting for wind speed using a modified EMD-based artificial neural network model. Renew Energy 2012;37:241-9. doi:10.1016/j.renene.2011.06.023.

[30] Liu H, Tian H, Liang X, Li Y. Wind speed forecasting approach using secondary decomposition algorithm and Elman neural networks. Appl Energy 2015;157:183-94. doi:10.1016/j.apenergy.2015.08.014.

[31] Zhang W, Qu Z, Zhang K, Mao W, Ma Y, Fan X. A combined model based on CEEMDAN and modified flower pollination algorithm for wind speed forecasting. Energy Convers Manag 2017;136:439-51. doi:10.1016/j.enconman.2017.01.022.

[32] Zhang Y, Liu K, Qin L, An X. Deterministic and probabilistic interval prediction for short-term wind power generation based on variational mode decomposition and machine learning methods. Energy Convers Manag 2016;112:208-19. doi:10.1016/j.enconman.2016.01.023.

[33] Liu H, Tian HQ, Chen C, Li YF. An experimental investigation of two Wavelet-MLP hybrid frameworks for wind speed prediction using GA and PSO optimization. Int $J$ Electr Power Energy Syst 2013;52:161-73. doi:10.1016/j.ijepes.2013.03.034.

[34] Su Z, Wang J, Lu H, Zhao G. A new hybrid model optimized by an intelligent 
optimization algorithm for wind speed forecasting. Energy Convers Manag 2014;85:443-52. doi:10.1016/j.enconman.2014.05.058.

[35] Wang S, Zhang N, Wu L, Wang Y. Wind speed forecasting based on the hybrid ensemble empirical mode decomposition and GA-BP neural network method. Renew Energy 2016;94:629 - 36. doi:10.1016/j.renene.2016.03.103.

[36] Liu H, Tian H, Liang X, Li Y. New wind speed forecasting approaches using fast ensemble empirical model decomposition, genetic algorithm, Mind Evolutionary Algorithm and Artificial Neural Networks. Renew Energy 2015;83:1066-75. doi:10.1016/j.renene.2015.06.004.

[37] Liu D, Niu D, Wang H, Fan L. Short-term wind speed forecasting using wavelet transform and support vector machines optimized by genetic algorithm. Renew Energy 2014;62:592-7. doi:10.1016/j.renene.2013.08.011.

[38] Catalao JPS, Pousinho HMI, Mendes VMF. Hybrid Wavelet-PSO-ANFIS Approach for Short-TermWind Power Forecasting in Portugal. IEEE Trans Sustain Energy 2010;2:50-9. doi:10.1109/TSTE.2010.2076359.

[39] Yang L, He M, Zhang J, Vittal V. Support-vector-machine-enhanced markov model for short-term wind power forecast. IEEE Trans Sustain Energy 2015;6:791-9. doi:10.1109/TSTE.2015.2406814.

[40]Lahouar A, Ben HadjSlama J. Hour-ahead wind power forecast based on random forests. Renew Energy 2017;109:529-41. doi:10.1016/j.renene.2017.03.064.

[41] Zhao Y, Ye L, Li Z, Song X, Lang Y, Su J. A novel bidirectional mechanism based on time series model for wind power forecasting. Appl Energy 2016;177:793-803. doi:10.1016/j.apenergy.2016.03.096.

[42] Dong Q, Sun Y, Li P. A novel forecasting model based on a hybrid processing strategy and an optimized local linear fuzzy neural network to make wind power forecasting: A case study of wind farms in China. Renew Energy 2017;102:241-57. doi:10.1016/j.renene.2016.10.030.

[43] Dong Q, Sun Y, Li P. A novel forecasting model based on a hybrid processing strategy and an optimized local linear fuzzy neural network to make wind power forecasting: A case study of wind farms in China. Renew Energy 2017;102:241-57. doi:10.1016/j.renene.2016.10.030.

[44] Sideratos G, Hatziargyriou ND. Probabilistic wind power forecasting using radial basis function neural networks. IEEE Trans Power Syst 2012;27:1788-96. doi:10.1109/TPWRS.2012.2187803.

[45] Chitsaz H, Amjady N, Zareipour H. Wind power forecast using wavelet neural network trained by improved Clonal selection algorithm. Energy Convers Manag 2015;89:588-98. doi:10.1016/j.enconman.2014.10.001.

[46] Wang HZ, Wang GB, Li GQ, Peng JC, Liu YT. Deep belief network based deterministic and probabilistic wind speed forecasting approach. Appl Energy 2016;182:80 - 93. doi:10.1016/j.apenergy.2016.08.108.

[47]Bremnes JB. Probabilistic wind power forecasts using local quantile regression. Wind Energy 2004;7:47-54. doi:10.1002/we.107.

[48] Nielsen HA, Madsen H, Nielsen TS. Using quanti le regression to extend an existing wind power forecasting system With probabilistic forecasts. Wind Energy, vol. 9, 2006, p. 95 - 108. doi:10.1002/we.180.

[49] Errouissi R, Cardenas-Barrera J, Meng J, Castillo-Guerra E, Gong X, Chang L. Bootstrap prediction interval estimation for wind speed forecasting. 2015 IEEE Energy Convers. Congr. Expo. ECCE 2015, 2015, p. 1919-24. doi:10.1109/ECCE.2015.7309931.

[50] Juban J, Siebert N, Kariniotakis GN. Probabilistic Short-term Wind Power 
Forecasting for the Optimal Management of Wind Generation. 2007 IEEE Lausanne Power Tech 2007:683-8. doi:10.1109/PCT.2007.4538398.

[51] Khosravi A, Nahavandi S, Creighton D, Atiya AF. Lower upper bound estimation method for construction of neural network-based prediction intervals. IEEE Trans Neural Networks 2011;22:337-46. doi:10.1109/TNN.2010.2096824.

[52] Yan J, Liu Y, Han S, Wang Y, Feng S. Reviews on uncertainty analysis of wind power forecasting. Renew Sustain Energy Rev 2015;52:1322-30. doi:10.1016/j.rser.2015.07.197.

[53] Bootstrapping and Resampling. http://www.ncl.ucar.edu/Applications/bootstrap.shtml.

[54] Yeh J-R, Shieh J-S, Huang NE. Complementary Ensemble Empirical Mode Decomposition: a Novel Noise Enhanced Data Analysis Method. Adv Adapt Data Anal 2010;02:135-56. doi:10.1142/S1793536910000422.

[55] Torres ME, Colominas MA, Schlotthauer G, Flandrin P. A complete ensemble empirical mode decomposition with adaptive noise. 2011 IEEE IntConfAcoust Speech Signal Process 2011;7:4144 - 7. doi:10.1109/ICASSP.2011.5947265.

[56] Huang NE, Shen Z, Long SR, Wu MC, Shih HH, Zheng Q, et al. The empirical mode decomposition and the Hilbert spectrum for nonlinear and non-stationary time series analysis. Proc R Soc A Math PhysEngSci 1998;454:903-95. doi:10.1098/rspa.1998.0193.

[57] Wu Z, Huang NE. Ensemble Empirical Mode Decomposition: a Noise-Assisted Data Analysis Method. Adv Adapt Data Anal 2009;01:1 - 41. doi:10.1142/S1793536909000047.

[58] Marwan N, Carmen Romano M, Thiel M, Kurths J. Recurrence plots for the analysis of complex systems. Phys Rep 2007;438:237-329. doi:10.1016/j.physrep.2006.11.001.

[59] Eckmann JP, Kamphorst SO, Ruelle D, Ciliberto S. Liapunov exponents from time series. Phys Rev A 1986;34:4971-9. doi:10.1103/PhysRevA.34.4971.

[60] Marwan N, Wessel N, Meyerfeldt U, Schirdewan A, Kurths J. Recurrence-plot-based measures of complexity and their application to heart-rate-variability data. Phys Rev E - Stat Nonlinear, Soft Matter Phys 2002;66. doi:10.1103/PhysRevE.66.026702.

[61] Zbilut JP, Webber CL. Embeddings and delays as derived from quantification of recurrence plots. PhysLett A 1992;171:199-203. doi:10.1016/0375-9601(92)90426-M.

[62] Webber CL, Zbilut JP. Dynamical assessment of physiological systems and states using recurrence plot strategies. J ApplPhysiol 1994;76:965 - 73.

[63] Kim HS, Eykholt R, Salas JD. Nonlinear dynamics, delay times, and embedding windows. Phys D Nonlinear Phenom 1999;127:48 - 60. doi:10.1016/S0167-2789(98)00240-1.

[64] Carbonneau R, Laframboise K, Vahidov R. Application of machine learning techniques for supply chain demand forecasting. Eur J Oper Res 2008;184:1140-54. doi:10.1016/j.ejor.2006.12.004.

[65] Vapnik VN. The Nature of Statistical Learning Theory. Springer 1995;8:188. doi:10.1109/TNN.1997.641482.

[66] Bouhouche S, LaksirYazid L, Hocine S, Bast J. Evaluation using online support-vector-machines and fuzzy reasoning. Application to condition monitoring of speeds rolling process. Control EngPract 2010;18:1060-8. doi:10.1016/j.conengprac.2010.05.010. 
[67] Wang JZ, Wang Y, Jiang P. The study and application of a novel hybrid forecasting model - A case study of wind speed forecasting in China. Appl Energy 2015;143:472 - 88. doi:10.1016/j.apenergy.2015.01.038.

[68] Han Z, Liu Y, Zhao J, Wang W. Real time prediction for converter gas tank levels based on multi-output least square support vector regressor. Control EngPract 2012;20:1400 - 9. doi:10.1016/j.conengprac.2012.08.006.

[69] Heidari AA, Ali Abbaspour R, RezaeeJordehi A. Gaussian bare-bones water cycle algorithm for optimal reactive power dispatch in electrical power systems. Appl Soft Comput J 2017;57. doi:10.1016/j.asoc.2017.04.048.

[70] Sarvi M, Avanaki IN. An optimized Fuzzy Logic Controller by Water Cycle Algorithm for power management of Stand-alone Hybrid Green Power generation. Energy Convers Manag 2015;106:118-26. doi:10.1016/j.enconman.2015.09.021.

[71] Gao K, Zhang Y, Sadollah A, Lentzakis A, Su R. Jaya, harmony search and water cycle algorithms for solving large-scale real-life urban traffic light scheduling problem. Swarm EvolComput 2016. doi:10.1016/j.swevo.2017.05.002.

[72] Eskandar H, Sadollah A, Bahreininejad A, Hamdi M. Water cycle algorithm - A novel metaheuristic optimization method for solving constrained engineering optimization problems. ComputStruct 2012;110-111:151-66. doi:10.1016/j.compstruc.2012.07.010.

[73] Khodabakhshian A, Esmaili MR, Bornapour M. Optimal coordinated design of UPFC and PSS for improving power system performance by using multi-objective water cycle algorithm. Int J Electr Power Energy Syst 2016;83:124-33. doi:10.1016/j.ijepes.2016.03.052.

[74] Sadollah A, Eskandar H, Kim JH. Water cycle algorithm for solving constrained multi-objective optimization problems. Appl Soft Comput 2015;27:279-98. doi:10.1016/j.asoc.2014.10.042.

[75] Deihimi A, KeshavarzZahed B, Iravani R. An interactive operation management of a micro-grid with multiple distributed generations using multi-objective uniform water cycle algorithm. Energy 2016;106:482-509. doi:10.1016/j.energy.2016.03.048.

[76] Deb K, Pratap A, Agarwal S, Meyarivan T. A fast and elitist multiobjective genetic algorithm: NSGA-II. IEEE Trans EvolComput 2002;6:182 - 97. doi:10.1109/4235.996017.

[77] Zitzler E, Laumanns M, Thiele L. SPEA2: Improving the Strength Pareto Evolutionary Algorithm. Evol Methods Des Optim Control with Appl to IndProbl 2001:95-100. doi:10.1.1.28.7571.

[78] Knowles J, Corne D. The Pareto archived evolution strategy: A new baseline algorithm for Pareto multiobjectiveoptimisation. Proc. 1999 Congr. Evol. Comput. CEC 1999, vol. 1, 1999, p. 98 - 105. doi:10.1109/CEC.1999.781913.

[79] Srinivas N, Deb K. Muiltiobjective Optimization Using Nondominated Sorting in Genetic Algorithms. EvolComput 1994;2:221-48. doi:10.1162/evco.1994.2.3.221.

[80] Zitzler E, Thiele L. Multiobjective Optimization Using Evolutionary Algorithms - A Comparative Case Study. ProcIntConf Parallel Probl Solving from Nat 1998:292 - 304. doi:10.1007/BFb0056872.

[81] Van Veldhuizen D a, Lamont GB. Evolutionary Computation and Convergence to a Pareto Front. Late Break Pap Genet Program 1998 Conf 1998:221-8.

[82] Hong YY, Lin JK. Interactive multi-objective active power scheduling considering uncertain renewable energies using adaptive chaos clonal evolutionary programming. Energy 2013;53:212-20. doi:10.1016/j.energy.2013.02.070.

[83] Schott JR, OH AIRFIOFTW-PAFB. Fault Tolerant Design Using Single and 
Multicriteria Genetic Algorithm Optimization. 1995, 37(1):1-13.

[84] Conti S, Nicolosi R, Rizzo SA, Zeineldin HH. Optimal dispatching of distributed generators and storage systems for MV islanded microgrids. IEEE Trans Power Deliv 2012;27:1243-51. doi:10.1109/TPWRD.2012.2194514.

[85] Wolf A, Swift JB, Swinney HL, Vastano JA. Determining Lyapunov exponents from a time series. Phys D Nonlinear Phenom 1985;16:285 - 317. doi:10.1016/0167-2789(85)90011-9.

[86] James G, Witten D, Hastie T, Tibshirani R. An Introduction to Statistical Learning. Economica 2013; 103(2):78 - 129. doi:10.1007/978-1-4614-7138-7.

[87] Bermingham ML, Pong-Wong R, Spiliopoulou a, Hayward C, Rudan I, Campbell $\mathrm{H}$, et al. Application of high-dimensional feature selection: evaluation for genomic prediction in man. Sci Rep 2015;5:10312. doi:10.1038/srep10312.

[88] Wang J, Du P, Niu T, Yang W. A novel hybrid system based on a new proposed algorithm-Multi-ObjectiveWhale Optimization Algorithm for wind speed forecasting. Appl Energy 2017. http://dx.doi.org/10.1016/j.apenergy.2017.10.031.

[89] Sun S, Qiao H, Wei Y, Wang S. A new dynamic integrated approach for wind speed forecasting. Appl Energy 2017;197:151-62. doi:10.1016/j.apenergy.2017.04.008. 